\title{
The permutation group method for the dilogarithm
}

\author{
GeORGES RHin AND CARLO Viola
}

\begin{abstract}
We give qualitative and quantitative improvements on all the best previously known irrationality results for dilogarithms of positive rational numbers. We obtain such improvements by applying our permutation group method to the diophantine study of double integrals of rational functions related to the dilogarithm.
\end{abstract}

Mathematics Subject Classification (2000): 11J82 (primary); 33B30, 20B35 (secondary).

\section{Introduction}

1.1 For $k \geq 1$ integer, the polylogarithm $\operatorname{Li}_{k}(x)$ of order $k$ is defined, in the unit disc $|x|<1$, by the power series

$$
\operatorname{Li}_{k}(x)=\sum_{n=1}^{\infty} \frac{x^{n}}{n^{k}}
$$

In particular

$$
\mathrm{Li}_{1}(x)=\sum_{n=1}^{\infty} \frac{x^{n}}{n}=-\log (1-x)
$$

and

$$
\operatorname{Li}_{2}(x)=\sum_{n=1}^{\infty} \frac{x^{n}}{n^{2}}=-\int_{0}^{x} \frac{\log (1-t)}{t} \mathrm{~d} t .
$$

The polylogarithm, which arises in Euler's work, is a typical instance of $G$-function. Following Siegel ([6], erster Teil, Section 4, VII), a $G$-function is defined to be a Taylor series $\sum a_{n} x^{n}$ whose coefficients are algebraic numbers such that, for a positive constant $C$ independent of $n$, (i) $a_{n}$ and its conjugates do not exceed $C^{n}$, and (ii) there is a common denominator for the coefficients $a_{v}$ with $v \leq n$ which does not exceed $C^{n}$.

Pervenuto alla Redazione il 4 novembre 2004 e in forma definitiva il 27 luglio 2005. 
As a special case of arithmetical study of such functions, we mention that rational approximations to values of the dilogarithm $\operatorname{Li}_{2}(x)$ at points $x=1 / s$ with $s \in \mathbb{Z}$ were studied by Hata [3], who proved that $\mathrm{Li}_{2}(1 / s)$ is irrational for all integers $s \in(-\infty,-5] \cup[7,+\infty)$, and gave irrationality measures of $\operatorname{Li}_{2}(1 / s)$ for the integers $s$ satisfying $7 \leq s \leq 18$ or $-16 \leq s \leq-5$. We refer to the introduction of the paper [3] for an account of earlier results concerning irrationality properties of $\operatorname{Li}_{2}(1 / s)$.

More generally, the broad subject of the irrationality or transcendence of values of $G$-functions was widely studied, mainly in a paper by Bombieri [2], where the author introduces the concept of Fuchsian differential operator of arithmetic type. In the spirit of Siegel's original method for $E$-functions [6], Bombieri makes a deep study of $G$-functions satisfying Fuchsian differential equations of arithmetic type. In particular ([2], Theorem 8), he obtains new algebraic independence results for polylogarithms of suitable algebraic numbers whose heights are large enough, and whose absolute values are sufficiently small, with an upper bound depending on the heights. Thus, for polylogarithms of rational numbers $r / s$, the hypotheses of Theorem 8 in [2] imply that $s$ is sufficiently large with respect to $r$, similarly to our assumptions in the present paper (see below). It is also worth mentioning that in the introduction to the paper [2] Bombieri remarks that an approach alternative to Siegel's for the arithmetical study of values of special $G$-functions at rational points can be obtained by explicit construction of Padé approximations to the functions involved.

In this paper we use a method for the diophantine study of dilogarithms of positive rational numbers which is independent of both Bombieri's and Padé approximation methods. We apply the permutation group method introduced by the authors in [4] and developed in [5] with the purpose of obtaining the best known irrationality measures of $\zeta(2)=\mathrm{Li}_{2}(1)=\pi^{2} / 6$ and $\zeta(3)=\mathrm{Li}_{3}(1)$. Our method allows us to prove a general result (Theorem 5.2) yielding the irrationality of $\operatorname{Li}_{2}(r / s)$ and an irrationality measure of this number under suitable quantitative conditions. Moreover, it provides an effective algorithm, described in Section 6, to compute, for any integer $r \geq 1$, a constant $s_{1}(r)>r$ such that for any integer $s \geq s_{1}(r)$ we have $\operatorname{Li}_{2}(r / s) \notin \mathbb{Q}$, with an explicit irrationality measure of $\operatorname{Li}_{2}(r / s)$ depending on $r$ but not on $s$.

In the diophantine study of $\mathrm{Li}_{2}(r / s)$, the restriction to the case $r=1$ made by Hata [3] is unnecessary for us. We also point out that the extension of our method to the study of dilogarithms of negative rational numbers would require no new ideas, nor would it entail really new difficulties, except for complications concerning the asymptotic study of some double complex integrals. In order to avoid a lengthy treatment of this technical point, in the present paper we prefer to confine ourselves to the case of dilogarithms of positive rational numbers.

Concerning $\mathrm{Li}_{2}(1 / s)$ for positive integers $s$, we extend Hata's results indicated above by proving in Section 6 that $\operatorname{Li}_{2}(1 / 6)$ is irrational, with irrationality measure

$$
\mu\left(\operatorname{Li}_{2}(1 / 6)\right)<783.29036 \ldots
$$


and we improve all the irrationality measures of $\mathrm{Li}_{2}(1 / s)$ given by Hata for $7 \leq$ $s \leq 18$ (see [3], p. 386, Table 1). As usual, we denote by $\mu(\theta)$ the least irrationality measure of an irrational number $\theta$, i.e., the least exponent $\mu$ such that for any $\varepsilon>0$ there exists a constant $q_{0}=q_{0}(\varepsilon)>0$ for which

$$
\left|\theta-\frac{p}{q}\right|>q^{-\mu-\varepsilon}
$$

for all integers $p$ and $q$ with $q>q_{0}$.

We give further applications of our method by proving that $\operatorname{Li}_{2}(2 / s) \notin \mathbb{Q}$ for $s \geq 51, \operatorname{Li}_{2}(3 / s) \notin \mathbb{Q}$ for $s \geq 173$ and $\operatorname{Li}_{2}(4 / s) \notin \mathbb{Q}$ for $s \geq 423$, together with some irrationality measures in these cases.

1.2 The main ideas and the common features in the various applications of our permutation group method, in the present paper as well as in our previous papers [4] and [5], can be roughly described as follows. Let $\theta$ be a constant related to integrals of rational functions, in the sense that, for a certain dimension $D$ depending on $\theta$,

$$
\theta=\int_{(0,1)^{D}} \frac{\mathrm{d} x_{1} \cdots \mathrm{d} x_{D}}{\Delta\left(x_{1}, \ldots, x_{D}\right)}
$$

where $\Delta\left(x_{1}, \ldots, x_{D}\right)$ is a suitable polynomial with rational coefficients (we have $D=2$ for $\theta=\zeta(2)$ and $D=3$ for $\theta=\zeta(3)$, and the above integral representations with $\Delta(x, y)=1-x y$ for $\zeta(2)$ and $\Delta(x, y, z)=2(1-(1-x y) z)$ for $\zeta(3)$ were introduced by Beukers in [1]). For the study of diophantine properties of $\theta$ we consider a suitable family of integrals

$$
\int_{(0,1)^{D}}\left(\frac{x_{1}^{a_{1}}\left(1-x_{1}\right)^{b_{1}} \cdots x_{D}^{a_{D}}\left(1-x_{D}\right)^{b_{D}}}{\Delta\left(x_{1}, \ldots, x_{D}\right)^{c}}\right)^{n} \frac{\mathrm{d} x_{1} \cdots \mathrm{d} x_{D}}{\Delta\left(x_{1}, \ldots, x_{D}\right)}
$$

where the exponents $a_{1}, \ldots, a_{D} ; b_{1}, \ldots, b_{D} ; c$ are integers, in general different from one another (for $\zeta(2)$ and $\zeta(3)$, the study of the case where such exponents are all equal is also due to Beukers [1]).

Being optimistic, one hopes that the integrals (1.1), possibly under suitable constraints for the exponents, represent linear forms in 1 and $\theta$ with rational coefficients (this is indeed the case for $\zeta(2)$ and $\zeta(3)$ ), and moreover that, for fixed $a_{1}, \ldots, a_{D} ; b_{1}, \ldots, b_{D} ; c$ and for $n \rightarrow \infty$, the corresponding linear form tends to zero rapidly enough, in comparison with the growth of its coefficients and of their denominators, to yield a good irrationality measure of $\theta$.

The core of our method consists in the construction of a finite group of birational transformations of dimension $D$ acting on the family of integrals (1.1). Combining the one-dimensional Euler integral representation of the hypergeometric function with the above transformation group, we obtain a permutation group acting on the set of exponents appearing in the integrals of the family. The algebraic structure of this permutation group yields strong information on the $p$-adic 
valuation of the coefficients of the linear form in 1 and $\theta$ given by the integral (1.1) for suitably fixed $a_{1}, \ldots, a_{D} ; b_{1}, \ldots, b_{D} ; c$, so that, by removing common prime factors of such coefficients, one can significantly improve the irrationality measure of $\theta$ that can be obtained from (1.1) by purely analytic methods. In some cases, e.g. for $\theta=\mathrm{Li}_{2}(1 / 6)$, the elimination of common prime factors of the coefficients provided by our permutation group allows us to prove the irrationality of $\theta$, a result that cannot be obtained by any other known method.

The application of the general strategy outlined above to the study of $\operatorname{Li}_{2}(r / s)$, which we carry out in the present paper, is naturally related with our method for $\zeta(2)=\mathrm{Li}_{2}(1)$ given in [4], but requires some essential modifications and involves some new difficulties. As in the case of $\zeta(2)$, the integrals of type (1.1) for $\operatorname{Li}_{2}(r / s)$ have dimension $D=2$; however the polynomial $\Delta(x, y)=x(1-y)+y z$ now appearing in the denominator contains a parameter $z>1$, subsequently chosen to be $z=s / r$, and is no longer a symmetric function of $x$ and $y$ as it was for $\zeta(2)$. As a consequence, we are forced to employ in our integrals a birational transformation (i.e., the involution $\lambda$ defined by (2.5) below) which transforms only the variable $y$ and acts identically on $x$, with an (inevitable) lack of symmetry, with respect to the case of $\zeta(2)$, in the resulting permutation group. Moreover, the integrals (1.1) over the unit square $(0,1)^{2}$, up to some controlled factors, yield linear forms not only in 1 and $\operatorname{Li}_{2}(1 / z)$, but also containing logarithmic terms involving $\log z$ and $\operatorname{Li}_{1}(1 / z)$. In order to get rid of these, as we do in Theorem 2.1, we have to treat simultaneously both double integrals of type $(1.1)$ over $(0,1)^{2}$, defined in $(2.1)$ below, and double integrals of mixed type, i.e. over the real interval $(0,1)$ in $x$ and over a complex contour in $y$, defined in (2.2). Also, similarly to our paper [4], the double contour integrals defined in (2.3) yield the coefficient of $\mathrm{Li}_{2}(1 / z)$ in the linear forms.

ACKNOWLEDGEMENT. We are indebted to the referee for helpful comments and suggestions on an earlier version of this paper.

\section{Linear forms involving $\mathrm{Li}_{1}$ and $\mathrm{Li}_{2}$}

Let $z \in \mathbb{R}, z>1$. For integer parameters $h, j, k, l, m \geq 0$ we define

$$
\begin{aligned}
& I_{z}^{(0)}(h, j, k, l, m)=z^{-l-m} \int_{0}^{1} \int_{0}^{1} \frac{x^{j}(1-x)^{h} y^{k}(1-y)^{l}}{(x(1-y)+y z)^{j+k-m+1}} \mathrm{~d} x \mathrm{~d} y, \\
& I_{z}^{(1)}(h, j, k, l, m) \\
& \quad=z^{-l-m} \int_{0}^{1}\left(\frac{1}{2 \pi i} \oint_{\left|y-\frac{x}{x-z}\right|=\varrho} \frac{x^{j}(1-x)^{h} y^{k}(1-y)^{l}}{(x(1-y)+y z)^{j+k-m+1}} \mathrm{~d} y\right) \mathrm{d} x,
\end{aligned}
$$




$$
\begin{aligned}
& I_{z}^{(2)}(h, j, k, l, m) \\
& \quad=z^{-l-m} \frac{1}{2 \pi i} \oint_{|x-z|=\sigma}\left(\frac{1}{2 \pi i} \oint_{\left|y-\frac{x}{x-z}\right|=\varrho} \frac{x^{j}(1-x)^{h} y^{k}(1-y)^{l}}{(x(1-y)+y z)^{j+k-m+1}} \mathrm{~d} y\right) \mathrm{d} x
\end{aligned}
$$

for any $\varrho, \sigma>0$, and

$$
I_{z}(h, j, k, l, m)=I_{z}^{(0)}(h, j, k, l, m)-(\log z) I_{z}^{(1)}(h, j, k, l, m) .
$$

Changing $x$ into $1-x$ and $y$ into $1-y$ in the integral (2.1) yields

$$
I_{z}^{(0)}(h, j, k, l, m)=z^{-l-m} \int_{0}^{1} \int_{0}^{1} \frac{x^{h}(1-x)^{j} y^{l}(1-y)^{k}}{(z-(z-1) y-x y)^{j+k-m+1}} \mathrm{~d} x \mathrm{~d} y .
$$

Hence in the limit case $z=1$ we have

$$
I_{1}^{(0)}(h, j, k, l, m)=I(h, j, k, l, m),
$$

where $I(h, j, k, l, m)$ is the integral, related to $\zeta(2)$, defined through the notation of the paper [4].

Here we are interested in the case $z>1$, which we assume. For $x \neq 0, x \neq z$, we consider the involution $\lambda=\lambda_{x, z}: y \mapsto Y$ defined by the equation

$$
\lambda: Y=\frac{1-y}{1-\frac{x-z}{x} y}=\frac{x(1-y)}{x(1-y)+y z},
$$

which satisfies

$$
\frac{\mathrm{d} Y}{x(1-Y)+Y z}=-\frac{\mathrm{d} y}{x(1-y)+y z} .
$$

Clearly for any $x>0$ the involution $\lambda$ maps the interval $0 \leq y \leq 1$ onto $0 \leq Y \leq 1$. Hence the change of variable (2.5) in (2.1) transforms the double integral over the unit square $(0,1)^{2}$ into a double integral over $(0,1)^{2}$. Also, for any fixed complex number $\tau \neq 0,1, \infty$, the function $Y=(1-y) /(1-\tau y)$ of the complex variable $y$ plainly transforms any circumference centred at $1 / \tau$ into a circumference centred at $1 / \tau$. Therefore, (2.5) transforms the contour integral over $|y-x /(x-z)|=\varrho$ appearing in (2.2) and (2.3) into a contour integral over $|Y-x /(x-z)|=\varrho^{\prime}$, for a suitable $\varrho^{\prime}>0$.

Consequently, if we apply the transformation $\lambda$ to the above integrals, i.e., if we make the change of variable (2.5), we easily obtain, owing to the normalization factor $z^{-l-m}$ appearing in (2.1), (2.2) and (2.3),

$$
I_{z}^{(v)}(h, j, k, l, m)=I_{z}^{(v)}(h, m, l, k, j) \quad(v=0,1,2),
$$


whence, by (2.4),

$$
I_{z}(h, j, k, l, m)=I_{z}(h, m, l, k, j) .
$$

Thus with the action of the transformation $\lambda$ on (2.1), (2.2), (2.3) and (2.4) we associate the permutation $\lambda$ of the set $\{h, j, k, l, m\}$ mapping $h, j, k, l, m$ respectively to $h, m, l, k, j$, i.e., given by

$$
\lambda=\left(\begin{array}{ll}
j & m
\end{array}\right)\left(\begin{array}{ll}
k & l
\end{array}\right),
$$

and the values of (2.1), (2.2), (2.3) and (2.4) are invariant under the action of $\lambda$.

As usual, for any integer $n \geq 1$ we define

$$
d_{n}=1 . \mathrm{c} \cdot \mathrm{m} .\{1, \ldots, n\},
$$

and $d_{0}=1$. Moreover, let

$$
\begin{aligned}
H & =\max \{l+m-j, m+h-k, h+j-l, j+k-m\}, \\
K & =\max \{l+m-j, \min \{m+h-k, h+j-l\}, j+k-m\}, \\
\alpha & =\max \{j+k, k+l, l+m\}, \\
\beta & =\max \{0, k+l-h\}, \\
\delta & =\max \{h, m+h-k, h+j-l, j+k, k+l, l+m\} .
\end{aligned}
$$

By linearity, we extend the action of the permutation $\lambda$ on any linear combination of $h, j, k, l, m$ with integer coefficients. Thus $\lambda(j+k)=\lambda(j)+\lambda(k)=l+m$, $\lambda(l+m-j)=\lambda(l)+\lambda(m)-\lambda(j)=j+k-m$, etc. We remark that $l+m-j$ and $j+k-m$ cannot be both $<0$, because $(l+m-j)+(j+k-m)=k+l \geq 0$. Hence the integers $H, K, \alpha, \beta, \delta$ defined by (2.9) are all non-negative and clearly invariant under the action of $\lambda$.

Theorem 2.1. With the above notation and assumptions we have

$$
d_{H} d_{K} z^{\alpha}(z-1)^{\beta} I_{z}(h, j, k, l, m)=P(z)-Q(z) \operatorname{Li}_{2}(1 / z)
$$

and

$$
d_{H} d_{K} z^{\alpha}(z-1)^{\beta} I_{z}^{(1)}(h, j, k, l, m)=R(z)-Q(z) \operatorname{Li}_{1}(1 / z),
$$

where

$$
P(z), Q(z), R(z) \in \mathbb{Z}[z], \quad \max \{\operatorname{deg} P(z), \operatorname{deg} Q(z), \operatorname{deg} R(z)\} \leq \delta .
$$

Moreover, the polynomial $Q(z)$ is given by

$$
Q(z)=d_{H} d_{K} z^{\alpha}(z-1)^{\beta} I_{z}^{(2)}(h, j, k, l, m) .
$$

The proof of Theorem 2.1 is based on some arithmetical lemmas. 
Lemma 2.2. If

$$
\min \{l+m-j, j+k-m\}<0
$$

then Theorem 2.1 holds with $Q(z)=R(z)=0$.

Proof. If $j+k-m<0$, the function

$$
\frac{x^{j}(1-x)^{h} y^{k}(1-y)^{l}}{(x(1-y)+y z)^{j+k-m+1}}
$$

is a polynomial $S(x, y, z) \in \mathbb{Z}[x, y, z]$. Therefore

$$
\oint_{\left|y-\frac{x}{x-z}\right|=\varrho} S(x, y, z) \mathrm{d} y=0,
$$

whence

$$
I_{z}^{(1)}(h, j, k, l, m)=I_{z}^{(2)}(h, j, k, l, m)=0 .
$$

We have

$$
\begin{aligned}
& \operatorname{deg}_{x} S(x, y, z)=m+h-k-1, \\
& \operatorname{deg}_{y} S(x, y, z)=l+m-j-1, \\
& \operatorname{deg}_{z} S(x, y, z)=m-j-k-1 .
\end{aligned}
$$

Consequently

$$
\begin{aligned}
& d_{m+h-k} d_{l+m-j} z^{l+m} I_{z}(h, j, k, l, m) \\
& \quad=d_{H^{\prime}} d_{K^{\prime}} \int_{0}^{1} \int_{0}^{1} S(x, y, z) \mathrm{d} x \mathrm{~d} y=T(z) \in \mathbb{Z}[z],
\end{aligned}
$$

where, by (2.9),

$$
\begin{aligned}
H^{\prime} & :=\max \{l+m-j, m+h-k\} \leq H, \\
K^{\prime} & :=\min \{l+m-j, m+h-k\} \leq K,
\end{aligned}
$$

so that $d_{H} / d_{H^{\prime}}$ and $d_{K} / d_{K^{\prime}}$ are integers, and $\operatorname{deg} T(z) \leq m-j-k-1$. Since $j+k-m<0$, we now have $k \leq j+k<m \leq l+m$, whence $k+l<l+m$, $j+k<l+m, \alpha=l+m$. It follows that

$$
d_{H} d_{K} z^{\alpha}(z-1)^{\beta} I_{z}(h, j, k, l, m)=P(z) \in \mathbb{Z}[z],
$$


with

$$
\begin{aligned}
& \operatorname{deg} P(z)=\beta+ \operatorname{deg} T(z) \leq \beta+m-j-k-1 \\
& \quad=\max \{m-j-k-1, l+m-h-j-1\}<l+m \leq \delta .
\end{aligned}
$$

This proves the lemma in the case $j+k-m<0$.

If $l+m-j<0$, the lemma follows from the invariance of $I_{z}(h, j, k, l, m)$, $I_{z}^{(1)}(h, j, k, l, m), I_{z}^{(2)}(h, j, k, l, m)$ and of the integers (2.9) under the action of $\lambda$, and from the previous argument for $j+k-m<0$.

Lemma 2.3. We have

$$
\begin{aligned}
I_{z}(0,0,0,0,0) & =\mathrm{Li}_{2}(1 / z), \\
I_{z}^{(1)}(0,0,0,0,0) & =\operatorname{Li}_{1}(1 / z), \\
I_{z}^{(2)}(0,0,0,0,0) & =-1,
\end{aligned}
$$

so that Theorem 2.1 holds for $h=j=k=l=m=0$.

Proof. We have

$$
\frac{1}{2 \pi i} \oint_{\left|y-\frac{x}{x-z}\right|=\varrho} \frac{\mathrm{d} y}{x(1-y)+y z}=\frac{1}{z-x},
$$

whence

$$
I_{z}^{(1)}(0,0,0,0,0)=\int_{0}^{1} \frac{\mathrm{d} x}{z-x}=-\log (1-1 / z)=\mathrm{Li}_{1}(1 / z)
$$

and

$$
I_{z}^{(2)}(0,0,0,0,0)=\frac{1}{2 \pi i} \oint_{|x-z|=\sigma} \frac{\mathrm{d} x}{z-x}=-1
$$

Since

$$
\int_{0}^{1} \frac{\mathrm{d} y}{x(1-y)+y z}=\frac{\log z-\log x}{z-x}
$$

we get

$$
I_{z}^{(0)}(0,0,0,0,0)=\int_{0}^{1} \int_{0}^{1} \frac{\mathrm{d} x \mathrm{~d} y}{x(1-y)+y z}=(\log z) \int_{0}^{1} \frac{\mathrm{d} x}{z-x}-\int_{0}^{1} \frac{\log x}{z-x} \mathrm{~d} x,
$$


whence, by (2.4) and (2.11),

$$
I_{z}(0,0,0,0,0)=-\int_{0}^{1} \frac{\log x}{z-x} \mathrm{~d} x
$$

With the change of variables $u=x / y, v=y$ we obtain

$$
\int_{0}^{1} \int_{0}^{1} \frac{\mathrm{d} u \mathrm{~d} v}{z-u v}=\int_{0}^{1} \frac{\mathrm{d} x}{z-x} \int_{x}^{1} \frac{\mathrm{d} y}{y}=-\int_{0}^{1} \frac{\log x}{z-x} \mathrm{~d} x .
$$

On the other hand

$$
\begin{aligned}
\int_{0}^{1} \int_{0}^{1} \frac{\mathrm{d} u \mathrm{~d} v}{z-u v}=\frac{1}{z} \int_{0}^{1} \int_{0}^{1} \sum_{r=0}^{\infty} \frac{u^{r} v^{r}}{z^{r}} \mathrm{~d} u \mathrm{~d} v \\
=\sum_{r=0}^{\infty} \frac{1}{z^{r+1}} \int_{0}^{1} u^{r} \mathrm{~d} u \int_{0}^{1} v^{r} \mathrm{~d} v=\sum_{n=1}^{\infty} \frac{1}{z^{n} n^{2}}=\operatorname{Li}_{2}(1 / z) .
\end{aligned}
$$

Therefore

$$
-\int_{0}^{1} \frac{\log x}{z-x} \mathrm{~d} x=\operatorname{Li}_{2}(1 / z)
$$

From (2.13) and (2.14) we obtain

$$
I_{z}(0,0,0,0,0)=\operatorname{Li}_{2}(1 / z)
$$

Lemma 2.4. If $h=j=l=m=0$ then Theorem 2.1 holds, with $Q(z)=0$ if $k>0$.

Proof. If $k=0$ we apply Lemma 2.3, so we may assume $k>0$. By Cauchy's integral formula we have

$$
\frac{1}{2 \pi i} \oint_{\left|y-\frac{x}{x-z}\right|=\varrho} \frac{y^{k}}{(x(1-y)+y z)^{k+1}} \mathrm{~d} y=\frac{1}{(z-x)^{k+1}},
$$


whence

$$
I_{z}^{(2)}(0,0, k, 0,0)=\frac{1}{2 \pi i} \oint_{|x-z|=\sigma} \frac{\mathrm{d} x}{(z-x)^{k+1}}=0
$$

and

$$
I_{z}^{(1)}(0,0, k, 0,0)=\int_{0}^{1} \frac{\mathrm{d} x}{(z-x)^{k+1}}=\frac{1}{k}\left(\frac{1}{(z-1)^{k}}-\frac{1}{z^{k}}\right) .
$$

Therefore

$$
k z^{k}(z-1)^{k} I_{z}^{(1)}(0,0, k, 0,0)=z^{k}-(z-1)^{k} .
$$

By partial integration we obtain

$$
\int_{0}^{1} \frac{y^{k}}{(x(1-y)+y z)^{k+1}} d y=-\frac{1}{k z^{k}(z-x)}+\frac{1}{z-x} \int_{0}^{1} \frac{y^{k-1}}{(x(1-y)+y z)^{k}} d y .
$$

On applying this formula recursively, we get

$$
\int_{0}^{1} \frac{y^{k}}{(x(1-y)+y z)^{k+1}} d y=-\sum_{r=0}^{k-1} \frac{1}{(k-r) z^{k-r}(z-x)^{r+1}}+\frac{\log z-\log x}{(z-x)^{k+1}} .
$$

Thus

$$
\begin{aligned}
& I_{z}^{(0)}(0,0, k, 0,0)=-\sum_{r=0}^{k-1} \frac{1}{(k-r) z^{k-r}} \int_{0}^{1} \frac{\mathrm{d} x}{(z-x)^{r+1}} \\
& +(\log z) \int_{0}^{1} \frac{\mathrm{d} x}{(z-x)^{k+1}}-\int_{0}^{1} \frac{\log x}{(z-x)^{k+1}} \mathrm{~d} x,
\end{aligned}
$$

whence, by (2.4) and (2.16),

$$
I_{z}(0,0, k, 0,0)=-\sum_{r=0}^{k-1} \frac{1}{(k-r) z^{k-r}} \int_{0}^{1} \frac{\mathrm{d} x}{(z-x)^{r+1}}-\int_{0}^{1} \frac{\log x}{(z-x)^{k+1}} \mathrm{~d} x .
$$


By partial integration we find

$$
\begin{aligned}
-\int_{0}^{1} & \frac{\log x}{(z-x)^{k+1}} \mathrm{~d} x=-\frac{1}{k} \int_{0}^{1}(\log x) \frac{\mathrm{d}}{\mathrm{d} x}\left((z-x)^{-k}-z^{-k}\right) \mathrm{d} x \\
= & \frac{1}{k} \int_{0}^{1}\left(\frac{1}{(z-x)^{k}}-\frac{1}{z^{k}}\right) \frac{\mathrm{d} x}{x}=\frac{1}{k} \int_{0}^{1} \frac{1}{z^{k}(z-x)^{k}} \frac{z^{k}-(z-x)^{k}}{z-(z-x)} \mathrm{d} x \\
= & \frac{1}{k} \sum_{r=0}^{k-1} \int_{0}^{1} \frac{z^{r}(z-x)^{k-1-r}}{z^{k}(z-x)^{k}} \mathrm{~d} x=\sum_{r=0}^{k-1} \frac{1}{k z^{k-r}} \int_{0}^{1} \frac{\mathrm{d} x}{(z-x)^{r+1}} .
\end{aligned}
$$

Therefore, by (2.18),

$$
\begin{aligned}
& I_{z}(0,0, k, 0,0)=\sum_{r=0}^{k-1}\left(\frac{1}{k}-\frac{1}{k-r}\right) \frac{1}{z^{k-r}} \int_{0}^{1} \frac{\mathrm{d} x}{(z-x)^{r+1}}= \\
& -\sum_{r=1}^{k-1} \frac{r}{k(k-r)} \frac{1}{z^{k-r}} \frac{1}{r}\left(\frac{1}{(z-1)^{r}}-\frac{1}{z^{r}}\right)=-\frac{1}{k z^{k}} \sum_{r=1}^{k-1} \frac{1}{k-r}\left(\frac{z^{r}}{(z-1)^{r}}-1\right) .
\end{aligned}
$$

We conclude that

$$
d_{k-1} k z^{k}(z-1)^{k-1} I_{z}(0,0, k, 0,0)=S(z) \in \mathbb{Z}[z],
$$

with $\operatorname{deg} S(z) \leq k-1$. This, together with (2.15) and (2.17), proves the lemma, since in the present case we have $H=K=\alpha=\beta=\delta=k$.

Lemma 2.5. If $h=j=k=m=0$ then Theorem 2.1 holds, with $Q(z)=0$ if $l>0$.

Proof. If $l=0$ we apply Lemma 2.3. If $l>0$ the result follows from Lemma 2.4 by applying the permutation $\lambda$.

Lemma 2.6. If $h=j=m=0$ then Theorem 2.1 holds, with $Q(z)=0$ unless $k=l=0$.

Proof. We may assume $k l>0$, for otherwise we apply Lemmas 2.3, 2.4 or 2.5. We have

$$
\int_{0}^{1} \frac{\mathrm{d} x}{(x(1-y)+y z)^{k+1}}=-\frac{1}{k(1-y)}\left(\frac{1}{(1+y(z-1))^{k}}-\frac{1}{y^{k} z^{k}}\right) .
$$


Hence

$$
\begin{gathered}
I_{z}^{(0)}(0,0, k, l, 0)=z^{-l} \int_{0}^{1} \int_{0}^{1} \frac{y^{k}(1-y)^{l}}{(x(1-y)+y z)^{k+1}} \mathrm{~d} x \mathrm{~d} y \\
=-\frac{1}{k z^{l}} \int_{0}^{1} \frac{y^{k}(1-y)^{l-1}}{(1+y(z-1))^{k}} \mathrm{~d} y+\frac{1}{k l z^{k+l}} .
\end{gathered}
$$

With the change of variable $y=(w-1) /(z-1)$ we get

$$
\begin{array}{r}
-\frac{1}{k z^{l}} \int_{0}^{1} \frac{y^{k}(1-y)^{l-1}}{(1+y(z-1))^{k}} \mathrm{~d} y=-\frac{1}{k z^{l}(z-1)^{k+l}} \int_{1}^{z} \frac{(w-1)^{k}(z-w)^{l-1}}{w^{k}} \mathrm{~d} w \\
\quad=-\frac{1}{k(z-1)^{k+l}} \sum_{r=0}^{k} \sum_{s=0}^{l-1}(-1)^{k-r-s}\left(\begin{array}{c}
k \\
r
\end{array}\right)\left(\begin{array}{c}
l-1 \\
s
\end{array}\right) \frac{1}{z^{s+1}} \int_{1}^{z} w^{r+s-k} \mathrm{~d} w .
\end{array}
$$

It follows that

$$
\begin{aligned}
& I_{z}^{(0)}(0,0, k, l, 0)=\frac{\log z}{k(z-1)^{k+l}} \sum_{\substack{r=0 \\
r+s=k-1}}^{k} \sum_{s=0}^{l-1}\left(\begin{array}{c}
k \\
r
\end{array}\right)\left(\begin{array}{c}
l-1 \\
s
\end{array}\right) \frac{1}{z^{s+1}} \\
& \quad-\frac{1}{k(z-1)^{k+l}} \sum_{\substack{r=0 \\
r+s \neq k-1}}^{k} \sum_{\substack{s=0 \\
r+s-1}}^{l-1)^{k-r-s}}\left(\begin{array}{c}
k \\
r+s+1
\end{array}\right)\left(\begin{array}{c}
l-1 \\
s
\end{array}\right)\left(\frac{1}{z^{k-r}}-\frac{1}{z^{s+1}}\right) \\
& \quad+\frac{1}{k l z^{k+l}} .
\end{aligned}
$$

A similar computation yields the values of $I_{z}^{(v)}(0,0, k, l, 0)$ for $v=1,2$. Let $\gamma$ denote a fixed contour in $\mathbb{C}$ enclosing the interval $\left[(1-z)^{-1}, 0\right]$ on the real axis. Then $\gamma$ encloses the point $x /(x-z)$ for any $x$ such that $0 \leq x \leq 1$. Therefore, by (2.2) and (2.20),

$$
\begin{aligned}
I_{z}^{(1)}(0,0, k, l, 0) & =z^{-l} \int_{0}^{1}\left(\frac{1}{2 \pi i} \oint_{\gamma} \frac{y^{k}(1-y)^{l}}{(x(1-y)+y z)^{k+1}} \mathrm{~d} y\right) \mathrm{d} x \\
& =-\frac{1}{k z^{l}} \frac{1}{2 \pi i} \oint_{\gamma} y^{k}(1-y)^{l-1}\left(\frac{1}{(1+y(z-1))^{k}}-\frac{1}{y^{k} z^{k}}\right) \mathrm{d} y \\
& =-\frac{1}{k z^{l}} \frac{1}{2 \pi i} \oint_{\gamma} \frac{y^{k}(1-y)^{l-1}}{(1+y(z-1))^{k}} \mathrm{~d} y .
\end{aligned}
$$


We apply again the change of variable $y=(w-1) /(z-1)$, which transforms $\gamma$ into a contour $\gamma^{\prime}$ enclosing the interval $[0,1]$ in the $w$-plane. Similarly to the previous case we obtain

$$
\begin{aligned}
& I_{z}^{(1)}(0,0, k, l, 0) \\
& \quad=-\frac{1}{k(z-1)^{k+l}} \sum_{r=0}^{k} \sum_{s=0}^{l-1}(-1)^{k-r-s}\left(\begin{array}{c}
k \\
r
\end{array}\right)\left(\begin{array}{c}
l-1 \\
s
\end{array}\right) \frac{1}{z^{s+1}} \frac{1}{2 \pi i} \oint_{\gamma^{\prime}} w^{r+s-k} \mathrm{~d} w,
\end{aligned}
$$

whence

$$
\begin{aligned}
I_{z}^{(1)}(0,0, k, l, 0) & =\frac{1}{k(z-1)^{k+l}} \sum_{\substack{r=0 \\
r+s=k-1}}^{k} \sum_{s=0}^{l-1}\left(\begin{array}{c}
k \\
r
\end{array}\right)\left(\begin{array}{c}
l-1 \\
s
\end{array}\right) \frac{1}{z^{s+1}} \\
& =\frac{1}{k(z-1)^{k+l}} \sum_{t=1}^{\min \{k, l\}}\left(\begin{array}{c}
k \\
k-t
\end{array}\right)\left(\begin{array}{l}
l-1 \\
t-1
\end{array}\right) \frac{1}{z^{t}} .
\end{aligned}
$$

Therefore

$$
k z^{\min \{k, l\}}(z-1)^{k+l} I_{z}^{(1)}(0,0, k, l, 0)=S(z) \in \mathbb{Z}[z]
$$

with $\operatorname{deg} S(z)=\min \{k, l\}-1$. Since in the present case we have

$$
H=K=\max \{k, l\}, \quad \alpha=\beta=\delta=k+l,
$$

we get

$$
d_{H} d_{K} z^{\alpha}(z-1)^{\beta} I_{z}^{(1)}(0,0, k, l, 0)=R(z) \in \mathbb{Z}[z],
$$

with $\operatorname{deg} R(z)=k+l-1<\delta$. From (2.4), (2.21) and (2.22) we obtain

$$
\begin{aligned}
& I_{z}(0,0, k, l, 0) \\
= & -\frac{1}{k(z-1)^{k+l}} \sum_{\substack{r=0 \\
r+s \neq k-1}}^{k} \sum_{\substack{s=0 \\
r+1}}^{l-1} \frac{(-1)^{k-r-s}}{r+k+1}\left(\begin{array}{c}
k \\
r
\end{array}\right)\left(\begin{array}{c}
l-1 \\
s
\end{array}\right)\left(\frac{1}{z^{k-r}}-\frac{1}{z^{s+1}}\right)+\frac{1}{k l z^{k+l}},
\end{aligned}
$$

whence

$$
d_{\max \{k-1, l\}} k z^{k+l}(z-1)^{k+l} I_{z}(0,0, k, l, 0)=T(z) \in \mathbb{Z}[z]
$$

with $\operatorname{deg} T(z) \leq k+l=\delta$. Consequently

$$
d_{H} d_{K} z^{\alpha}(z-1)^{\beta} I_{z}(0,0, k, l, 0)=P(z) \in \mathbb{Z}[z]
$$

with $\operatorname{deg} P(z)=\operatorname{deg} T(z) \leq \delta$. 
We have to compute

$$
\begin{array}{r}
I_{z}^{(2)}(0,0, k, l, 0) \\
\quad=z^{-l} \frac{1}{2 \pi i} \oint_{|x-z|=\sigma}\left(\frac{1}{2 \pi i} \oint_{\left|y-\frac{x}{x-z}\right|=\varrho} \frac{y^{k}(1-y)^{l}}{(x(1-y)+y z)^{k+1}} \mathrm{~d} y\right) \mathrm{d} x .
\end{array}
$$

Note that $|x-z|=\sigma$ if and only if $|x /(x-z)-1|=z / \sigma$. Hence the contour $|y-x /(x-z)|=\varrho$ in (2.23) can be replaced by $|y-1|=\varrho$ provided that $\varrho \sigma>z$, since under this assumption the circumference $|y-1|=\varrho$ encloses the point $x /(x-z)$ for any $x$ satisfying $|x-z|=\sigma$. After this replacement, we may interchange the integrations over $|x-z|=\sigma$ and $|y-1|=\varrho$. It follows that

$$
\begin{aligned}
& I_{z}^{(2)}(0,0, k, l, 0) \\
= & z^{-l} \frac{1}{2 \pi i} \oint_{|y-1|=\varrho} y^{k}(1-y)^{l}\left(\frac{1}{2 \pi i} \oint_{\left|x-\frac{y z}{y-1}\right|=\sigma} \frac{\mathrm{d} x}{(x(1-y)+y z)^{k+1}}\right) \mathrm{d} y,
\end{aligned}
$$

again for any $\varrho, \sigma>0$. The inner integral equals

$$
\frac{1}{(1-y)^{k+1}} \oint_{\left|x-\frac{y z}{y-1}\right|=\sigma} \frac{\mathrm{d} x}{\left(x-\frac{y z}{y-1}\right)^{k+1}}=0,
$$

since $k>0$. We conclude that $I_{z}^{(2)}(0,0, k, l, 0)=0$, and the lemma follows.

Lemma 2.7. If $k=l=0$ and $m=j$, then Theorem 2.1 holds.

Proof. From (2.12) we get

$$
\begin{aligned}
z^{j} I_{z}^{(0)}(h, j, 0,0, j) & =\int_{0}^{1} \int_{0}^{1} \frac{x^{j}(1-x)^{h}}{x(1-y)+y z} \mathrm{~d} x \mathrm{~d} y=\int_{0}^{1} x^{j}(1-x)^{h} \frac{\log z-\log x}{z-x} \mathrm{~d} x \\
& =(\log z) \int_{0}^{1} \frac{x^{j}(1-x)^{h}}{z-x} \mathrm{~d} x-\int_{0}^{1} \frac{x^{j}(1-x)^{h}}{z-x} \log x \mathrm{~d} x,
\end{aligned}
$$

and from (2.10)

$$
\begin{aligned}
z^{j} I_{z}^{(1)}(h, j, 0,0, j) & =\int_{0}^{1}\left(\frac{1}{2 \pi i} \oint_{\left|y-\frac{x}{x-z}\right|=\varrho} \frac{x^{j}(1-x)^{h}}{x(1-y)+y z} \mathrm{~d} y\right) \mathrm{d} x \\
& =\int_{0}^{1} \frac{x^{j}(1-x)^{h}}{z-x} \mathrm{~d} x .
\end{aligned}
$$


Therefore

$$
z^{j} I_{z}(h, j, 0,0, j)=-\int_{0}^{1} \frac{x^{j}(1-x)^{h}}{z-x} \log x \mathrm{~d} x
$$

We write $x=z-(z-x)$ and $1-x=(z-x)-(z-1)$. From (2.25) we obtain

$$
\begin{aligned}
& z^{j} I_{z}^{(1)}(h, j, 0,0, j) \\
& \quad=\sum_{r=0}^{j} \sum_{s=0}^{h}(-1)^{r+h-s}\left(\begin{array}{l}
j \\
r
\end{array}\right)\left(\begin{array}{l}
h \\
s
\end{array}\right) z^{j-r}(z-1)^{h-s} \int_{0}^{1}(z-x)^{r+s-1} \mathrm{~d} x .
\end{aligned}
$$

Splitting the double sum into $r=s=0$ and $(r, s) \neq(0,0)$ we get by $(2.11)$

$$
\begin{aligned}
& z^{j} I_{z}^{(1)}(h, j, 0,0, j)=(-1)^{h} z^{j}(z-1)^{h} \operatorname{Li}_{1}(1 / z) \\
& \quad+\sum_{\substack{r=0 \\
(r, s) \neq(0,0)}}^{j} \sum_{\substack{s=0 \\
h}}^{h}(-1)^{r+h-s}\left(\begin{array}{l}
j \\
r
\end{array}\right)\left(\begin{array}{l}
h \\
s
\end{array}\right) z^{j-r}(z-1)^{h-s} \frac{z^{r+s}-(z-1)^{r+s}}{r+s} .
\end{aligned}
$$

We now have $H=K=\delta=h+j, \alpha=j, \beta=0$. By (2.27) we obtain

$$
\begin{aligned}
d_{H} d_{K} z^{\alpha}(z-1)^{\beta} I_{z}^{(1)}(h, j, 0,0, j) & =d_{h+j}^{2} z^{j} I_{z}^{(1)}(h, j, 0,0, j) \\
& =R(z)-Q(z) \operatorname{Li}_{1}(1 / z),
\end{aligned}
$$

with $Q(z), R(z) \in \mathbb{Z}[z], \max \{\operatorname{deg} Q(z), \operatorname{deg} R(z)\}=h+j=\delta$, and

$$
Q(z)=(-1)^{h+1} d_{h+j}^{2} z^{j}(z-1)^{h} .
$$

Similarly, by (2.26),

$$
\begin{aligned}
& z^{j} I_{z}(h, j, 0,0, j) \\
& \quad=-\sum_{r=0}^{j} \sum_{s=0}^{h}(-1)^{r+h-s}\left(\begin{array}{l}
j \\
r
\end{array}\right)\left(\begin{array}{l}
h \\
s
\end{array}\right) z^{j-r}(z-1)^{h-s} \int_{0}^{1}(z-x)^{r+s-1} \log x \mathrm{~d} x .
\end{aligned}
$$

By (2.14), the contribution for $r=s=0$ equals

$$
-(-1)^{h} z^{j}(z-1)^{h} \int_{0}^{1} \frac{\log x}{z-x} \mathrm{~d} x=(-1)^{h} z^{j}(z-1)^{h} \operatorname{Li}_{2}(1 / z) .
$$


As in (2.19), for $(r, s) \neq(0,0)$ we get by partial integration

$$
\begin{aligned}
-\int_{0}^{1}(z-x)^{r+s-1} \log x \mathrm{~d} x & =\frac{1}{r+s} \int_{0}^{1} \frac{z^{r+s}-(z-x)^{r+s}}{z-(z-x)} \mathrm{d} x \\
& =\frac{1}{r+s} \sum_{t=1}^{r+s} z^{r+s-t} \int_{0}^{1}(z-x)^{t-1} \mathrm{~d} x \\
& =\frac{1}{r+s} \sum_{t=1}^{r+s} z^{r+s-t} \frac{z^{t}-(z-1)^{t}}{t} .
\end{aligned}
$$

It follows that

$$
\begin{aligned}
& z^{j} I_{z}(h, j, 0,0, j)=(-1)^{h} z^{j}(z-1)^{h} \operatorname{Li}_{2}(1 / z) \\
& +\sum_{\substack{r=0 \\
(r, s) \neq(0,0)}}^{j} \sum_{s=0}^{h}(-1)^{r+h-s}\left(\begin{array}{l}
j \\
r
\end{array}\right)\left(\begin{array}{l}
h \\
s
\end{array}\right) z^{j-r}(z-1)^{h-s} \\
& \times \frac{1}{r+s} \sum_{t=1}^{r+s} \frac{1}{t}\left(z^{r+s}-z^{r+s-t}(z-1)^{t}\right) .
\end{aligned}
$$

Hence

$$
\begin{aligned}
d_{H} d_{K} z^{\alpha}(z-1)^{\beta} I_{z}(h, j, 0,0, j) & =d_{h+j}^{2} z^{j} I_{z}(h, j, 0,0, j) \\
& =P(z)-Q(z) \operatorname{Li}_{2}(1 / z),
\end{aligned}
$$

with $Q(z)$ given by (2.28) and with $P(z) \in \mathbb{Z}[z], \operatorname{deg} P(z) \leq h+j=\delta$.

By (2.10) we get

$$
\begin{aligned}
z^{j} I_{z}^{(2)}(h, j, 0,0, j) & =\frac{1}{2 \pi i} \oint_{|x-z|=\sigma}\left(\frac{1}{2 \pi i} \oint_{\left|y-\frac{x}{x-z}\right|=\varrho} \frac{x^{j}(1-x)^{h}}{x(1-y)+y z} \mathrm{~d} y\right) \mathrm{d} x \\
& =\frac{1}{2 \pi i} \oint_{|x-z|=\sigma} \frac{x^{j}(1-x)^{h}}{z-x} \mathrm{~d} x \\
& =-\operatorname{Res}_{x=z} \frac{x^{j}(1-x)^{h}}{x-z}=-z^{j}(1-z)^{h},
\end{aligned}
$$

whence, by (2.28),

$$
\begin{aligned}
Q(z)=-d_{h+j}^{2} z^{j}(1-z)^{h} & =d_{h+j}^{2} z^{j} I_{z}^{(2)}(h, j, 0,0, j) \\
& =d_{H} d_{K} z^{\alpha}(z-1)^{\beta} I_{z}^{(2)}(h, j, 0,0, j) .
\end{aligned}
$$

This completes the proof of the lemma. 
Finally, we require the following lemma relating the values of $\alpha, \beta$ and $\delta$ in (2.9).

Lemma 2.8. If $\beta=k+l-h$, then $\delta=\alpha$.

If $\beta=0$ and $\alpha=j+k$, then $\delta=h+j-l$.

If $\beta=0$ and $\alpha=k+l$, then $\delta=h$.

If $\beta=0$ and $\alpha=l+m$, then $\delta=m+h-k$.

Proof. If $\beta=k+l-h$ we have $k+l-h \geq 0$, whence $h \leq k+l, m+h-k \leq l+m$ and $h+j-l \leq j+k$. Therefore

$$
\delta=\max \{j+k, k+l, l+m\}=\alpha .
$$

If $\beta=0$ we have $k+l-h \leq 0$, whence $k+l \leq h, l+m \leq m+h-k$ and $j+k \leq h+j-l$. Therefore

$$
\delta=\max \{h, m+h-k, h+j-l\} .
$$

If $\alpha=j+k$ then $k+l \leq j+k, l \leq j, h \leq h+j-l$, and $l+m \leq j+k$, $m+h-k \leq h+j-l$, whence $\delta=h+j-l$. If $\alpha=k+l$ then $j+k \leq k+l$, $j \leq l, h+j-l \leq h$, and $l+m \leq k+l, m \leq k, m+h-k \leq h$, whence $\delta=h$. If $\alpha=l+m$ then $k+l \leq l+m, k \leq m, h \leq m+h-k$, and $j+k \leq l+m$, $h+j-l \leq m+h-k$, whence $\delta=\bar{m}+h-\bar{k}$.

Proof of Theorem 2.1. If $\min \{l+m-j, j+k-m\}<0$ we apply Lemma 2.2. Thus we may assume $l+m-j \geq 0$ and $j+k-m \geq 0$. If $k m>0$, we use the linear decomposition of the integrals $I_{z}^{(v)}(h, j, k, l, m)(v=0,1,2)$ given by the identity $y=1-(1-y)$. We have

$$
\begin{aligned}
I_{z}^{(0)} & (h, j, k, l, m) \\
& =z^{-l-m} \int_{0}^{1} \int_{0}^{1} \frac{x^{j}(1-x)^{h} y^{k-1}(1-y)^{l}}{(x(1-y)+y z)^{j+(k-1)-(m-1)+1}}(1-(1-y)) \mathrm{d} x \mathrm{~d} y \\
& =z^{-1} I_{z}^{(0)}(h, j, k-1, l, m-1)-I_{z}^{(0)}(h, j, k-1, l+1, m-1),
\end{aligned}
$$

and similarly for $I_{z}^{(1)}(h, j, k, l, m)$ and $I_{z}^{(2)}(h, j, k, l, m)$. Hence the same decomposition holds also for $I_{z}(h, j, k, l, m)$ :

$$
I_{z}(h, j, k, l, m)=z^{-1} I_{z}(h, j, k-1, l, m-1)-I_{z}(h, j, k-1, l+1, m-1) .
$$

For brevity, denote the tuples on the right side of (2.29) by

$$
\begin{aligned}
& \left(h_{1}, j_{1}, k_{1}, l_{1}, m_{1}\right)=(h, j, k-1, l, m-1), \\
& \left(h_{2}, j_{2}, k_{2}, l_{2}, m_{2}\right)=(h, j, k-1, l+1, m-1) .
\end{aligned}
$$


If we denote by $H_{q}, K_{q}, \alpha_{q}, \beta_{q}, \delta_{q}$ the integers (2.9) associated with the tuple $\left(h_{q}, j_{q}, k_{q}, l_{q}, m_{q}\right)(q=1,2)$, we easily get

$$
\begin{aligned}
& H_{1} \leq H, \quad K_{1} \leq K, \quad \alpha_{1}=\alpha-1, \quad \beta-1 \leq \beta_{1} \leq \beta, \quad \delta-1 \leq \delta_{1} \leq \delta, \\
& H_{2} \leq H, \quad K_{2} \leq K, \quad \alpha-1 \leq \alpha_{2} \leq \alpha, \quad \beta_{2}=\beta, \quad \delta-1 \leq \delta_{2} \leq \delta .
\end{aligned}
$$

Since $H_{q} \leq H$ and $K_{q} \leq K$, the quotients $d_{H} / d_{H_{q}}$ and $d_{K} / d_{K_{q}}$ are integers. Hence if Theorem 2.1 holds for each of the tuples (2.30) we have, for $q=1,2$,

$$
\left\{\begin{array}{l}
d_{H} d_{K} z^{\alpha_{q}}(z-1)^{\beta_{q}} I_{z}\left(h_{q}, j_{q}, k_{q}, l_{q}, m_{q}\right)=P_{q}(z)-Q_{q}(z) \operatorname{Li}_{2}(1 / z) \\
d_{H} d_{K} z^{\alpha_{q}}(z-1)^{\beta_{q}} I_{z}^{(1)}\left(h_{q}, j_{q}, k_{q}, l_{q}, m_{q}\right)=R_{q}(z)-Q_{q}(z) \operatorname{Li}_{1}(1 / z) \\
d_{H} d_{K} z^{\alpha_{q}}(z-1)^{\beta_{q}} I_{z}^{(2)}\left(h_{q}, j_{q}, k_{q}, l_{q}, m_{q}\right)=Q_{q}(z)
\end{array}\right.
$$

with

$$
P_{q}(z), Q_{q}(z), R_{q}(z) \in \mathbb{Z}[z], \quad \max \left\{\operatorname{deg} P_{q}(z), \operatorname{deg} Q_{q}(z), \operatorname{deg} R_{q}(z)\right\} \leq \delta_{q} .
$$

From (2.29), (2.30), (2.31) and (2.32) we obtain

$$
\begin{aligned}
& d_{H} d_{K} z^{\alpha}(z-1)^{\beta} I_{z}(h, j, k, l, m) \\
& =d_{H} d_{K} z^{\alpha}(z-1)^{\beta}\left(z^{-1} I_{z}(h, j, k-1, l, m-1)-I_{z}(h, j, k-1, l+1, m-1)\right) \\
& =d_{H} d_{K} z^{\alpha_{1}+1}(z-1)^{\beta_{1}}(z-1)^{\beta-\beta_{1}} z^{-1} I_{z}\left(h_{1}, j_{1}, k_{1}, l_{1}, m_{1}\right) \\
& \quad-d_{H} d_{K} z^{\alpha-\alpha_{2}} z^{\alpha_{2}}(z-1)^{\beta_{2}} I_{z}\left(h_{2}, j_{2}, k_{2}, l_{2}, m_{2}\right) \\
& =(z-1)^{\beta-\beta_{1}}\left(P_{1}(z)-Q_{1}(z) \operatorname{Li}_{2}(1 / z)\right)-z^{\alpha-\alpha_{2}}\left(P_{2}(z)-Q_{2}(z) \operatorname{Li}_{2}(1 / z)\right) \\
& =P(z)-Q(z) \operatorname{Li}_{2}(1 / z),
\end{aligned}
$$

where

and

$$
P(z)=(z-1)^{\beta-\beta_{1}} P_{1}(z)-z^{\alpha-\alpha_{2}} P_{2}(z)
$$

$$
Q(z)=(z-1)^{\beta-\beta_{1}} Q_{1}(z)-z^{\alpha-\alpha_{2}} Q_{2}(z) .
$$

Similarly

$$
\begin{aligned}
& d_{H} d_{K} z^{\alpha}(z-1)^{\beta} I_{z}^{(1)}(h, j, k, l, m)=R(z)-Q(z) \operatorname{Li}_{1}(1 / z), \\
& d_{H} d_{K} z^{\alpha}(z-1)^{\beta} I_{z}^{(2)}(h, j, k, l, m)=Q(z),
\end{aligned}
$$

with

$$
R(z)=(z-1)^{\beta-\beta_{1}} R_{1}(z)-z^{\alpha-\alpha_{2}} R_{2}(z)
$$

and with $Q(z)$ given by (2.34). 
By (2.31) we have $\beta-\beta_{1} \geq 0$ and $\alpha-\alpha_{2} \geq 0$. Hence, by (2.33),

$$
P(z), Q(z), R(z) \in \mathbb{Z}[z],
$$

with

$$
\max \{\operatorname{deg} P(z), \operatorname{deg} Q(z), \operatorname{deg} R(z)\} \leq \max \left\{\beta-\beta_{1}+\delta_{1}, \alpha-\alpha_{2}+\delta_{2}\right\} .
$$

We claim that

$$
\max \left\{\beta-\beta_{1}+\delta_{1}, \alpha-\alpha_{2}+\delta_{2}\right\} \leq \delta .
$$

By (2.31) we know that $\beta_{1}$ is either $\beta$ or $\beta-1$. If $\beta_{1}=\beta$ we get

$$
\beta-\beta_{1}+\delta_{1}=\delta_{1} \leq \delta .
$$

If $\beta_{1}=\beta-1$, since $\beta=\max \{0, k+l-h\}$ and, by (2.30), $\beta_{1}=\max \{0, k-1+$ $l-h\}$, we get $\beta=k+l-h>0, \beta_{1}=k+l-h-1$, whence, by Lemma 2.8 and by (2.31), $\delta_{1}=\alpha_{1}=\alpha-1=\delta-1$. Therefore

$$
\beta-\beta_{1}+\delta_{1}=1+\delta-1=\delta .
$$

Similarly, again by (2.31), $\alpha_{2}$ is either $\alpha$ or $\alpha-1$. If $\alpha_{2}=\alpha$ then

$$
\alpha-\alpha_{2}+\delta_{2}=\delta_{2} \leq \delta .
$$

If $\alpha_{2}=\alpha-1$, since

$$
\alpha=\max \{j+k, k+l, l+m\}
$$

and, by (2.30),

$$
\alpha_{2}=\max \{j+k-1, k+l, l+m\},
$$

we have $\alpha=j+k, \alpha_{2}=j+k-1$. If $\beta=\beta_{2}=0$, by Lemma 2.8 we get $\delta=h+j-l, \delta_{2}=h+j-l-1=\delta-1$. If $\beta=\beta_{2}=k+l-h$, again by Lemma 2.8 we get $\delta_{2}=\alpha_{2}=\alpha-1=\delta-1$. In either case

$$
\alpha-\alpha_{2}+\delta_{2}=1+\delta-1=\delta .
$$

From (2.37), (2.38), (2.39) and (2.40) we deduce (2.36). From (2.35) and (2.36) we have

$$
\max \{\operatorname{deg} P(z), \operatorname{deg} Q(z), \operatorname{deg} R(z)\} \leq \delta,
$$

and we conclude that if Theorem 2.1 holds for $(h, j, k-1, l, m-1)$ and $(h, j, k-1, l+1, m-1)$, it holds also for $(h, j, k, l, m)$. 
If $k m=0$ but $j l>0$, we use the invariance of (2.2), (2.3), (2.4) and of the integers (2.9) under the action of the permutation $\lambda$, and then apply the linear decomposition (2.29). Therefore, if Theorem 2.1 holds for $(h, j-1, k, l-1, m)$ and $(h, j-1, k+1, l-1, m)$, it holds also for $(h, j, k, l, m)$.

If $h l>0$, we may use the linear decomposition of $I_{z}^{(v)}(h, j, k, l, m)(v=$ $0,1,2)$, and hence of $I_{z}(h, j, k, l, m)$, given by the identity

$$
(1-x)(1-y)=z-(z-1)(1-y)-(x(1-y)+y z) .
$$

Thus we obtain

$$
\begin{aligned}
& I_{z}(h, j, k, l, m)=I_{z}(h-1, j, k, l-1, m) \\
& \quad-(z-1) I_{z}(h-1, j, k, l, m)-I_{z}(h-1, j, k, l-1, m+1),
\end{aligned}
$$

and similarly for $I_{z}^{(v)}(h, j, k, l, m)$.

We denote the tuples on the right side of (2.41) by

$$
\begin{aligned}
& \left(h_{3}, j_{3}, k_{3}, l_{3}, m_{3}\right)=(h-1, j, k, l-1, m), \\
& \left(h_{4}, j_{4}, k_{4}, l_{4}, m_{4}\right)=(h-1, j, k, l, m), \\
& \left(h_{5}, j_{5}, k_{5}, l_{5}, m_{5}\right)=(h-1, j, k, l-1, m+1),
\end{aligned}
$$

and again by $H_{q}, K_{q}, \alpha_{q}, \beta_{q}, \delta_{q}$ the integers (2.9) associated with the tuple $\left(h_{q}, j_{q}, k_{q}, l_{q}, m_{q}\right)(q=3,4,5)$. We easily see that

$$
\begin{aligned}
& H_{3} \leq H, \quad K_{3} \leq K, \quad \alpha-1 \leq \alpha_{3} \leq \alpha, \quad \beta_{3}=\beta, \quad \delta-1 \leq \delta_{3} \leq \delta, \\
& H_{4} \leq H, \quad K_{4} \leq K, \quad \alpha_{4}=\alpha, \quad \beta \leq \beta_{4} \leq \beta+1, \quad \delta-1 \leq \delta_{4} \leq \delta, \\
& H_{5} \leq H, \quad K_{5} \leq K, \quad \alpha-1 \leq \alpha_{5} \leq \alpha, \quad \beta_{5}=\beta, \quad \delta-1 \leq \delta_{5} \leq \delta .
\end{aligned}
$$

Thus if Theorem 2.1 holds for each of the three tuples (2.42), we have (2.32) and (2.33) for $q=3,4,5$. Similarly to the previous case, by (2.41), (2.42) and (2.43) we get

$$
\begin{aligned}
& d_{H} d_{K} z^{\alpha}(z-1)^{\beta} I_{z}(h, j, k, l, m)=P(z)-Q(z) \operatorname{Li}_{2}(1 / z), \\
& d_{H} d_{K} z^{\alpha}(z-1)^{\beta} I_{z}^{(1)}(h, j, k, l, m)=R(z)-Q(z) \operatorname{Li}_{1}(1 / z), \\
& d_{H} d_{K} z^{\alpha}(z-1)^{\beta} I_{z}^{(2)}(h, j, k, l, m)=Q(z),
\end{aligned}
$$

with

$$
\begin{aligned}
& P(z)=z^{\alpha-\alpha_{3}} P_{3}(z)-(z-1)^{\beta+1-\beta_{4}} P_{4}(z)-z^{\alpha-\alpha_{5}} P_{5}(z), \\
& Q(z)=z^{\alpha-\alpha_{3}} Q_{3}(z)-(z-1)^{\beta+1-\beta_{4}} Q_{4}(z)-z^{\alpha-\alpha_{5}} Q_{5}(z), \\
& R(z)=z^{\alpha-\alpha_{3}} R_{3}(z)-(z-1)^{\beta+1-\beta_{4}} R_{4}(z)-z^{\alpha-\alpha_{5}} R_{5}(z) .
\end{aligned}
$$


By (2.43) we have $\alpha-\alpha_{3} \geq 0, \beta+1-\beta_{4} \geq 0$ and $\alpha-\alpha_{5} \geq 0$. Thus by (2.33) for $q=3,4,5$ we get

$$
P(z), Q(z), R(z) \in \mathbb{Z}[z]
$$

with

$\max \{\operatorname{deg} P(z), \operatorname{deg} Q(z), \operatorname{deg} R(z)\}$

$$
\leq \max \left\{\alpha-\alpha_{3}+\delta_{3}, \beta+1-\beta_{4}+\delta_{4}, \alpha-\alpha_{5}+\delta_{5}\right\} .
$$

A discussion analogous with the one given in the previous case, again based on Lemma 2.8 and on (2.43) for $\alpha_{q}, \beta_{q}, \delta_{q}(q=3,4,5)$, easily yields

$$
\max \left\{\alpha-\alpha_{3}+\delta_{3}, \beta+1-\beta_{4}+\delta_{4}, \alpha-\alpha_{5}+\delta_{5}\right\} \leq \delta,
$$

similarly to (2.36). From (2.44) and (2.45) we have

$$
\max \{\operatorname{deg} P(z), \operatorname{deg} Q(z), \operatorname{deg} R(z)\} \leq \delta,
$$

so that if Theorem 2.1 holds for $(h-1, j, k, l-1, m),(h-1, j, k, l, m)$ and $(h-1, j, k, l-1, m+1)$, it holds also for $(h, j, k, l, m)$.

If $h l=0$ but $h k>0$, we apply first the permutation $\lambda$ and then the linear decomposition (2.41). Hence if Theorem 2.1 holds for $(h-1, j, k-1, l, m)$, $(h-1, j, k, l, m)$ and $(h-1, j+1, k-1, l, m)$, it holds also for $(h, j, k, l, m)$.

With the notation (2.30) and (2.42), if for at least one $q(1 \leq q \leq 5)$ the tuple $\left(h_{q}, j_{q}, k_{q}, l_{q}, m_{q}\right)$ satisfies at least one of the inequalities $k_{q} m_{q}>0, j_{q} l_{q}>0$, $h_{q} l_{q}>0, h_{q} k_{q}>0$, we iterate the linear decompositions (2.29) or (2.41), up to applying the permutation $\lambda$. because

We remark that such an iteration process must terminate in finitely many steps,

$$
h+j+k+l+m>h_{q}+j_{q}+k_{q}+l_{q}+m_{q} \geq 0 \quad(q=1, \ldots, 5) .
$$

Hence if we iterate the decompositions (2.29) and (2.41) sufficiently many times, we end up with finitely many tuples for each of which either $\min \{l+m-j, j+$ $k-m\}<0$, in which case we apply Lemma 2.2, or $k m=j l=h l=h k=$ 0 . Thus Theorem 2.1 will be proved in general, if we show that it holds for any $(h, j, k, l, m)$ satisfying $k m=j l=h l=h k=0, l+m-j \geq 0, j+k-m \geq 0$. We distinguish some cases.

First case: $j>0$. From $j l=0$ we get $l=0$. Since $l+m-j \geq 0$ we have $m \geq j>0$, and from $k m=0$ we get $k=0$. Since $j+k-m \geq 0$ we have $j \geq m$. Therefore $m=j$. Thus we have the tuple $(h, j, 0,0, j)$, and we apply Lemma 2.7.

Second case: $j=0, k>0$. From $k m=h k=0$ we get $h=m=0$. Thus we have $(0,0, k, l, 0)$, and we apply Lemma 2.6 . 
Third case: $j=k=0, l>0$. From $h l=0$ we get $h=0$, and from $j+k-m \geq 0$ we get $m=0$. Thus we have $(0,0,0, l, 0)$, and we apply Lemma 2.5 .

Fourth case: $j=k=l=0, h>0$. Since $j+k-m \geq 0$ we have $m=0$. Thus we have $(h, 0,0,0,0)$, and again we apply Lemma 2.7 .

Fifth case: $j=k=l=h=0$. Again by $j+k-m \geq 0$ we get $m=0$. Thus we have $(0,0,0,0,0)$, and we apply Lemma 2.3 .

\section{The permutation group}

In this section we show that the integrals (2.1), (2.2), (2.3) and (2.4) for $z>1$ can be equipped with an algebraic structure, though not so rich as in the case $z=1$ treated in [4]. Our aim is to construct a permutation group acting on $h, j, k, l, m$ together with the integers $l+m-j, m+h-k, h+j-l, j+k-m$ occurring in (2.9), similarly to the construction in [4], and to derive the corresponding transformation formulae for the integrals (2.1), (2.2), (2.3) and (2.4).

Let

$$
\mathcal{S}=\{h, j, k, l, m, l+m-j, m+h-k, h+j-l, j+k-m\} .
$$

We recall from Section 2 that the permutation $\lambda$ given by (2.8) acts on the set $\mathcal{S}$ as follows:

$$
\lambda=(j m)(k l)(l+m-j j+k-m)(m+h-k h+j-l) .
$$

We now use the hypergeometric integral transformation, based on Euler's integral representation of the hypergeometric function. It is well known that for any $a, b, c \in \mathbb{C}$ satisfying $\operatorname{Re} c>\max \{\operatorname{Re} a, \operatorname{Re} b\}$ and $\min \{\operatorname{Re} a, \operatorname{Re} b\}>0$, and for any $t \in \mathbb{C} \backslash[1,+\infty)$, we have

$$
\int_{0}^{1} \frac{x^{b-1}(1-x)^{c-b-1}}{(1-x t)^{a}} \mathrm{~d} x=\frac{\Gamma(b) \Gamma(c-b)}{\Gamma(a) \Gamma(c-a)} \int_{0}^{1} \frac{x^{a-1}(1-x)^{c-a-1}}{(1-x t)^{b}} \mathrm{~d} x,
$$

where $\Gamma$ denotes the Euler gamma-function (see [4], formula (3.2)). Hence, choosing $a=j+k-m+1, b=j+1, c=h+j+2$, and $t=(y-1) /(y z)$ for $0<y \leq 1<z$, we get

$$
\begin{aligned}
& \int_{0}^{1} \frac{x^{j}(1-x)^{h}}{\left(1+x \frac{1-y}{y z}\right)^{j+k-m+1}} \mathrm{~d} x \\
& \quad=\frac{h ! j !}{(m+h-k) !(j+k-m) !} \int_{0}^{1} \frac{x^{j+k-m}(1-x)^{m+h-k}}{\left(1+x \frac{1-y}{y z}\right)^{j+1}} \mathrm{~d} x,
\end{aligned}
$$

provided $m+h-k \geq 0$ and $j+k-m \geq 0$. 
Multiplying (3.4) by

$$
z^{-l-m} \frac{y^{k}(1-y)^{l}}{(y z)^{j+k-m+1}}
$$

and integrating in $0 \leq y \leq 1$ we easily obtain

$$
\begin{aligned}
& I_{z}^{(0)}(h, j, k, l, m) \\
& \quad=\frac{h ! j !}{(m+h-k) !(j+k-m) !} I_{z}^{(0)}(m+h-k, j+k-m, m, l, k) .
\end{aligned}
$$

As for $I_{z}^{(1)}(h, j, k, l, m)$, let $\gamma$ denote a fixed contour in $\mathbb{C}$ enclosing the interval $\left[(1-z)^{-1}, 0\right]$ on the real axis. As in the proof of Lemma 2.6 we have

$$
I_{z}^{(1)}(h, j, k, l, m)=z^{-l-m} \frac{1}{2 \pi i} \oint_{\gamma} \frac{y^{k}(1-y)^{l}}{(y z)^{j+k-m+1}} \mathrm{~d} y \int_{0}^{1} \frac{x^{j}(1-x)^{h}}{\left(1+x \frac{1-y}{y z}\right)^{j+k-m+1}} \mathrm{~d} x,
$$

and using again (3.4) for $y \in \gamma$ we get

$$
\begin{aligned}
& I_{z}^{(1)}(h, j, k, l, m) \\
& \quad=\frac{h ! j !}{(m+h-k) !(j+k-m) !} I_{z}^{(1)}(m+h-k, j+k-m, m, l, k) .
\end{aligned}
$$

By (2.4), (3.5) and (3.6) we have

$$
\begin{aligned}
& I_{z}(h, j, k, l, m) \\
& \quad=\frac{h ! j !}{(m+h-k) !(j+k-m) !} I_{z}(m+h-k, j+k-m, m, l, k) .
\end{aligned}
$$

We now transform $I_{z}^{(2)}(h, j, k, l, m)$. Similarly to (2.24) we have

$$
\begin{aligned}
& I_{z}^{(2)}(h, j, k, l, m) \\
& =z^{-l-m} \frac{1}{2 \pi i} \oint_{|y-1|=\varrho}\left(\frac{1}{2 \pi i} \oint_{\left|x-\frac{y z}{y-1}\right|=\sigma} \frac{x^{j}(1-x)^{h} y^{k}(1-y)^{l}}{(x(1-y)+y z)^{j+k-m+1}} \mathrm{~d} x\right) \mathrm{d} y .
\end{aligned}
$$

We require the analogue of (3.3) for contour integrals and for integer exponents, which can be elementarily proved. 
Lemma 3.1. Let $a, b, c$ be integers satisfying $c>\max \{a, b\}$ and $\min \{a, b\}>0$. Let $t \in \mathbb{C}, t \neq 0$. Then, for any $\sigma>0$,

$$
\begin{aligned}
\frac{1}{2 \pi i} \oint_{|x-1 / t|=\sigma} & \frac{x^{b-1}(1-x)^{c-b-1}}{(1-x t)^{a}} \mathrm{~d} x \\
& =\frac{(b-1) !(c-b-1) !}{(a-1) !(c-a-1) !} \frac{1}{2 \pi i} \oint_{|x-1 / t|=\sigma} \frac{x^{a-1}(1-x)^{c-a-1}}{(1-x t)^{b}} \mathrm{~d} x .
\end{aligned}
$$

Proof. Using Cauchy's integral formula we have

$$
\begin{aligned}
\frac{1}{2 \pi i} \oint_{|x-1 / t|=\sigma} \frac{x^{b-1}(1-x)^{c-b-1}}{(1-x t)^{a}} \mathrm{~d} x=\left(-\frac{1}{t}\right)^{a} \frac{1}{2 \pi i} \oint_{|x-1 / t|=\sigma} \frac{x^{b-1}(1-x)^{c-b-1}}{(x-1 / t)^{a}} \mathrm{~d} x \\
=\left(-\frac{1}{t}\right)^{a} \frac{1}{(a-1) !}\left[\frac{\mathrm{d}^{a-1}}{\mathrm{~d} x^{a-1}}\left(x^{b-1}(1-x)^{c-b-1}\right)\right]_{x=1 / t} \\
=\left(-\frac{1}{t}\right)^{a} \frac{1}{(a-1) !}\left[\frac{\mathrm{d}^{a-1}}{\mathrm{~d} x^{a-1}} \sum_{r=0}^{c-b-1}(-1)^{r}\left(\begin{array}{c}
c-b-1 \\
r
\end{array}\right) x^{b-1+r}\right]_{x=1 / t} \\
=\left(-\frac{1}{t}\right)^{a} \sum_{r=\max \{0, a-b\}}^{c-b-1}(-1)^{r}\left(\begin{array}{c}
c-b-1 \\
r
\end{array}\right)\left(\begin{array}{c}
b-1+r \\
a-1
\end{array}\right) \frac{1}{t^{b-a+r}} \\
=(-1)^{a+b} \sum_{r=\max \{0, a-b\}}^{c-b-1}\left(\begin{array}{c}
c-b-1 \\
r
\end{array}\right)\left(\begin{array}{c}
b-1+r \\
a-1
\end{array}\right)\left(-\frac{1}{t}\right)^{b+r} .
\end{aligned}
$$

If we set $r=a-b+s$, the last sum becomes

$$
\begin{aligned}
& \sum_{s=\max \{0, b-a\}}^{c-a-1}\left(\begin{array}{c}
c-b-1 \\
a-b+s
\end{array}\right)\left(\begin{array}{c}
a-1+s \\
a-1
\end{array}\right)\left(-\frac{1}{t}\right)^{a+s} \\
= & \frac{(b-1) !(c-b-1) !}{(a-1) !(c-a-1) !} \sum_{s=\max \{0, b-a\}}^{c-a-1}\left(\begin{array}{c}
c-a-1 \\
s
\end{array}\right)\left(\begin{array}{c}
a-1+s \\
b-1
\end{array}\right)\left(-\frac{1}{t}\right)^{a+s} .
\end{aligned}
$$

Since the factor $(-1)^{a+b}$ is symmetric in $a$ and $b$, the lemma follows. 
From Lemma 3.1 we obtain, if $m+h-k \geq 0$ and $j+k-m \geq 0$,

$$
\begin{aligned}
& \frac{1}{2 \pi i} \oint_{\left|x-\frac{y z}{y-1}\right|=\sigma} \frac{x^{j}(1-x)^{h}}{\left(1+x \frac{1-y}{y z}\right)^{j+k-m+1}} \mathrm{~d} x \\
& =\frac{h ! j !}{(m+h-k) !(j+k-m) !} \frac{1}{2 \pi i} \oint_{\left|x-\frac{y z}{y-1}\right|=\sigma} \frac{x^{j+k-m}(1-x)^{m+h-k}}{\left(1+x \frac{1-y}{y z}\right)^{j+1}} \mathrm{~d} x .
\end{aligned}
$$

Multiplying by $z^{-l-m} y^{k}(1-y)^{l} /(y z)^{j+k-m+1}$ and integrating over $|y-1|=\varrho$ yields, by (3.8),

$$
\begin{aligned}
& I_{z}^{(2)}(h, j, k, l, m) \\
& \quad=\frac{h ! j !}{(m+h-k) !(j+k-m) !} I_{z}^{(2)}(m+h-k, j+k-m, m, l, k) .
\end{aligned}
$$

In order to simplify our notation, we denote by

$$
J_{z}(h, j, k, l, m)
$$

any one of the integrals (2.1), (2.2), (2.3) or (2.4). By (2.6), (2.7), (3.5), (3.6), (3.7) and (3.9) we have the transformation formulae

$$
J_{z}(h, j, k, l, m)=J_{z}(h, m, l, k, j)
$$

and, if $m+h-k \geq 0$ and $j+k-m \geq 0$,

$$
\begin{aligned}
& J_{z}(h, j, k, l, m) \\
& \quad=\frac{h ! j !}{(m+h-k) !(j+k-m) !} J_{z}(m+h-k, j+k-m, m, l, k) .
\end{aligned}
$$

From now on, we assume the non-negative integers $h, j, k, l, m$ to be chosen so that $l+m-j, m+h-k, h+j-l, j+k-m$ are also non-negative. The transformation formulae (3.10) and (3.11) can be written as

$$
\frac{J_{z}(h, j, k, l, m)}{h ! j ! k ! l ! m !}=\frac{J_{z}(h, m, l, k, j)}{h ! m ! l ! k ! j !}
$$

and

$$
\frac{J_{z}(h, j, k, l, m)}{h ! j ! k ! l ! m !}=\frac{J_{z}(m+h-k, j+k-m, m, l, k)}{(m+h-k) !(j+k-m) ! m ! l ! k !}
$$


respectively. Let $\varphi$ be the hypergeometric integral transformation acting on the quotient

$$
\frac{J_{z}(h, j, k, l, m)}{h ! j ! k ! l ! m !}
$$

as is given by (3.13). It is natural to associate with $\varphi$ the permutation $\varphi$ mapping $h$, $j, k, l, m$ respectively to $m+h-k, j+k-m, m, l, k$, and extended by linearity to any linear combination of $h, j, k, l, m$ with integer coefficients. Thus the action of the permutation $\varphi$ on the set $\mathcal{S}$ defined by (3.1) is the following:

$$
\boldsymbol{\varphi}=(h m+h-k)(j j+k-m)(k m) .
$$

Similarly, the transformation $\lambda$ defined by (2.5) acts on the quotient (3.14) as is given by (3.12), and we associate with $\lambda$ the permutation $\lambda$ whose action on $\mathcal{S}$ is given by (3.2).

We remark that both $\lambda$ and $\varphi$ act identically on the integer $k+l-h$, which occurs in the definition (2.9) of $\beta$. Hence $k+l-h$ can be excluded from the present discussion. Accordingly the sign of $k+l-h$ is immaterial, and we allow $k+l-h \gtreqless 0$.

As in [4] pp. 38-39, if we apply to (3.14) any product $\chi$ of transformations each of which is either $\varphi$ or $\lambda$, we plainly have

$$
\frac{J_{z}(h, j, k, l, m)}{h ! j ! k ! l ! m !}=\frac{J_{z}(\boldsymbol{\chi}(h), \chi(j), \chi(k), \chi(l), \chi(m))}{\boldsymbol{\chi}(h) ! \chi(j) ! \chi(k) ! \chi(l) ! \chi(m) !}
$$

where $\chi$ is the corresponding product of permutations $\varphi$ or $\lambda$ in reverse order. Thus the value of the quotient (3.14) is invariant under the action of the permutation group

$$
\Phi=\langle\varphi, \lambda\rangle
$$

generated by $\lambda$ and $\varphi$.

We have seen that $\boldsymbol{\Phi}$ acts on the set $\mathcal{S}$. Similarly to the papers [4] and [5], however, the structure of the group $\Phi$ is best analysed by considering the actions of $\lambda$ and $\varphi$ on the integers

$$
h+j, \quad j+k, k+l, \quad l+m, m+h,
$$

which, for brevity, we denote by $u_{1}, u_{2}, u_{3}, u_{4}, u_{5}$ respectively. From (3.2) and (3.15) we see that the actions of $\lambda$ and $\varphi$ on such integers are the following:

$$
\begin{aligned}
& \lambda=\left(\begin{array}{ll}
u_{1} & u_{5}
\end{array}\right)\left(\begin{array}{ll}
u_{2} & u_{4}
\end{array}\right), \\
& \varphi=\left(\begin{array}{ll}
u_{3} & u_{4}
\end{array}\right) .
\end{aligned}
$$


Moreover, if a permutation $\chi \in \boldsymbol{\Phi}=\langle\boldsymbol{\varphi}, \lambda\rangle$ acts identically on $u_{1}, \ldots, u_{5}$ we get by linearity

$$
\begin{aligned}
2 \chi(h) & =\chi(2 h)=\chi\left(u_{1}-u_{2}+u_{3}-u_{4}+u_{5}\right) \\
& =\chi\left(u_{1}\right)-\chi\left(u_{2}\right)+\chi\left(u_{3}\right)-\chi\left(u_{4}\right)+\chi\left(u_{5}\right) \\
& =u_{1}-u_{2}+u_{3}-u_{4}+u_{5}=2 h,
\end{aligned}
$$

and similarly $2 \chi(j)=2 j$, etc. Hence $\chi$ acts identically on $h, j, k, l, m$, and we infer that $\chi$ is the identity. This shows that the permutation group $\Phi$ acts faithfully on $\left\{u_{1}, \ldots, u_{5}\right\}$, and in particular $\boldsymbol{\Phi}$ is isomorphic to a subgroup of the symmetric group $\mathfrak{S}_{5}$.

It is plain from (3.17) that $\Phi=\langle\varphi, \lambda\rangle$ is intransitive over the set $\left\{u_{1}, \ldots, u_{5}\right\}$, since each of the two subsets $\left\{u_{1}, u_{5}\right\}$ and $\left\{u_{2}, u_{3}, u_{4}\right\}$ is mapped onto itself by both $\lambda$ and $\varphi$. Also, we easily see that $\varphi \lambda \varphi \lambda \varphi=\left(u_{2} u_{4}\right)$ whence $\varphi \lambda \varphi \lambda \varphi \lambda=\lambda \varphi \lambda \varphi \lambda \varphi=$ $\left(\begin{array}{ll}u_{1} & u_{5}\end{array}\right)$, and therefore

$$
\left(\begin{array}{ll}
u_{1} & u_{5}
\end{array}\right),\left(u_{2} u_{4}\right),\left(\begin{array}{ll}
u_{3} & u_{4}
\end{array}\right) \in \Phi .
$$

Thus $\boldsymbol{\Phi}$ is isomorphic to the product of the symmetric groups of permutations of $\left\{u_{1}, u_{5}\right\}$ and of $\left\{u_{2}, u_{3}, u_{4}\right\}$ :

$$
\Phi \cong \mathfrak{S}_{2} \times \mathfrak{S}_{3},
$$

whence its order is $|\Phi|=2 ! \cdot 3 !=12$.

Clearly $\Phi$ is intransitive also over the set $\mathcal{S}$, since the cardinality $|\mathcal{S}|=9$ does not divide the order $|\boldsymbol{\Phi}|=12$. In fact, each of the two subsets $\{h, m+h-k, h+$ $j-l\}$ and $\{j, k, l, m, l+m-j, j+k-m\}$ is mapped onto itself by both $\lambda$ and $\varphi$.

With any permutation $\chi \in \Phi$ we associate the quotient

$$
\frac{h ! j ! k ! l ! m !}{\chi(h) ! \chi(j) ! \chi(k) ! \chi(l) ! \chi(m) !}
$$

resulting from the transformation formula (3.16) for $J_{z}(h, j, k, l, m)$. Plainly if $\boldsymbol{\chi}, \boldsymbol{\chi}^{\prime} \in \boldsymbol{\Phi}$ lie in the same left coset of the subgroup

$$
\Lambda=\langle\lambda\rangle
$$

of order 2 in $\Phi$, i.e., if $\chi=\chi^{\prime} \chi^{\prime \prime}$ where $\chi^{\prime \prime}$ is either the identity or $\lambda$, the quotient (3.18) equals the analogous quotient with $\chi^{\prime}$ in place of $\chi$ (for any permutations $\chi_{1}$ and $\chi_{2}$, we denote by $\chi_{1} \chi_{2}$ the permutation obtained by applying first $\chi_{2}$ and then $\chi_{1}$ ). Therefore, with each left coset of $\boldsymbol{\Lambda}$ in $\boldsymbol{\Phi}$ we associate the corresponding quotient (3.18), where $\chi$ is either permutation lying in the coset considered.

For any $\chi \in \Phi$, we simplify the quotient (3.18) by removing the factorials of the integers appearing both in the numerator and in the denominator, i.e., lying in

$$
\{h, j, k, l, m\} \cap\{\boldsymbol{\chi}(h), \boldsymbol{\chi}(j), \boldsymbol{\chi}(k), \boldsymbol{\chi}(l), \boldsymbol{\chi}(m)\}
$$


independently of the numerical values assigned to $h, j, k, l, m$. If, after simplifying (3.18), the resulting quotient has $v$ factorials in the numerator and $v$ in the denominator, we say that $\chi$ is a permutation of level $v$, or that the left coset $\chi \Lambda$ is of level $v$. For instance, from the transformation formulae (3.10) and (3.11) we see that $\lambda$ and $\varphi$ are permutations of levels 0 and 2, respectively.

Since $|\boldsymbol{\Phi}|=12$ and $|\boldsymbol{\Lambda}|=2$, there are 6 left cosets of $\boldsymbol{\Lambda}$ in $\boldsymbol{\Phi}$, as follows:

$$
\begin{aligned}
& 1 \text { coset of level } 0, \\
& 3 \text { cosets of level } 2, \\
& 2 \text { cosets of level } 3 \text {. }
\end{aligned}
$$

The coset of level 0 is the subgroup $\Lambda$ itself. For each of the remaining 5 left cosets of $\Lambda$ in $\Phi$ we choose one of the two permutations lying in the coset, and write below the corresponding transformation formula (3.16) for $J_{z}(h, j, k, l, m)$, together with the permutation chosen.

$$
\begin{aligned}
J_{z}(h, j, k, l, m) & \\
= & \frac{h ! j !}{(m+h-k) !(j+k-m) !} J_{z}(m+h-k, j+k-m, m, l, k) \\
= & \frac{h ! m !}{(h+j-l) !(l+m-j) !} J_{z}(h+j-l, l+m-j, j, k, l) \\
= & \frac{k ! l !}{(l+m-j) !(j+k-m) !} J_{z}(h, j, l+m-j, j+k-m, m) \\
= & \frac{h ! j ! k !}{(h+j-l) !(l+m-j) !(j+k-m) !} \\
= & \frac{h ! l ! m !}{(m+h-k) !(j+k-m) !(l+m-j) !} \\
\quad & \quad \times J_{z}(m+h-k, j+k-m, l+m-j, j, k)
\end{aligned}
$$

\section{The arithmetical correction}

As we remarked, the integers $H, K, \alpha, \beta, \delta$ defined by (2.9) are invariant under the action of the permutation $\lambda$ given by (3.2), and it is easy to see that $\alpha, \beta, \delta$ are also invariant under the action of the permutation $\varphi$ in (3.15), while $H$ and $K$ are not. However, in order to apply the group-theoretic arguments developed in Section 3 we require, in place of $H$ and $K$, suitable integers $M$ and $N$ invariant under the action of the whole permutation group $\Phi=\langle\varphi, \lambda\rangle$. Thus we define

$$
M=\max \mathcal{S},
$$


where $\mathcal{S}$ is the set (3.1). Also, for the successive maxima in a sequence we use the notation $\max , \max ^{\prime}, \ldots$ if $\mathcal{A}=\left(a_{1}, \ldots, a_{n}\right)$ is any finite sequence of real numbers and $i_{1}, \ldots, i_{n}$ is a reordering of $1, \ldots, n$ such that $a_{i_{1}} \geq a_{i_{2}} \geq \ldots \geq a_{i_{n}}$, we set

$$
\max \mathcal{A}=a_{i_{1}}, \quad \max ^{\prime} \mathcal{A}=a_{i_{2}} .
$$

Then we define

$$
N=\max \left\{\max ^{\prime}(h, m+h-k, h+j-l), j, k, l, m, l+m-j, j+k-m\right\} .
$$

In other words, $N$ is the maximum in the sequence of the eight integers obtained by omitting from $\mathcal{S}$ the maximum of $h, m+h-k, h+j-l$ (with only one omission, even if ( $h, m+h-k, h+j-l)$ contains more than one maximal element).

The integers $M$ and $N$ are invariant under the action of the group $\Phi$ since, as we remarked in Section 3, $\boldsymbol{\Phi}$ is intransitive over $\mathcal{S}$ and maps the subset $\{h, m+$ $h-k, h+j-l\}$ onto itself. Also, $H$ and $K$ defined in (2.9) plainly do not exceed, respectively, $M$ and $N$ defined by (4.1) and (4.2). Therefore Theorem 2.1 holds a fortiori with $M$ in place of $H$ and $N$ in place of $K$. Moreover, by the invariance of $M, N, \alpha, \beta, \delta$ under the action of the whole group $\boldsymbol{\Phi}$ it follows that, for any $\chi \in \Phi$, Theorem 2.1 holds with $h, j, k, l, m$ respectively replaced by $\chi(h), \chi(j)$, $\chi(k), \boldsymbol{\chi}(l), \chi(m)$ and with polynomials $P_{\boldsymbol{\chi}}(z), Q_{\chi}(z), R_{\chi}(z) \in \mathbb{Z}[z]$ depending on the left coset $\chi \Lambda$, but with $M$ and $N$ in place of $H$ and $K$ respectively and with $\alpha, \beta, \delta$ all independent of $\chi$.

For $h, j, k, l, m$ fixed and $n=1,2, \ldots$, we replace the tuple $(h, j, k, l, m)$ in Theorem 2.1 by $(h n, j n, k n, l n, m n)$. Hence $M, N, \alpha, \beta, \delta$ are replaced by $M n$, $N n, \alpha n, \beta n, \delta n$ respectively. Also, we choose the number $z>1$ to be rational. We denote

$$
z=s / r \text { with } r, s \in \mathbb{Z}, 1 \leq r<s .
$$

Then Theorem 2.1 yields in particular

$$
\left\{\begin{aligned}
d_{M n} d_{N n}(s / r)^{\alpha n}(s / r-1)^{\beta n} I_{s / r} & (h n, j n, k n, l n, m n) \\
& =P_{n}(s / r)-Q_{n}(s / r) \operatorname{Li}_{2}(r / s) \\
d_{M n} d_{N n}(s / r)^{\alpha n}(s / r-1)^{\beta n} I_{s / r}^{(2)} & (h n, j n, k n, l n, m n)=Q_{n}(s / r),
\end{aligned}\right.
$$

where $P_{n}$ and $Q_{n}$ are polynomials with integer coefficients of degrees not exceeding $\delta n$. It follows that $r^{\delta n} P_{n}(s / r)$ and $r^{\delta n} Q_{n}(s / r)$ are integers. Thus, multiplying (4.3) by $r^{\delta n}$ we obtain

$$
\left\{\begin{array}{r}
d_{M n} d_{N n} s^{\alpha n}(s-r)^{\beta n} r^{(\delta-\alpha-\beta) n} I_{s / r}(h n, j n, k n, l n, m n) \\
=A_{n}-B_{n} \operatorname{Li}_{2}(r / s) \\
d_{M n} d_{N n} s^{\alpha n}(s-r)^{\beta n} r^{(\delta-\alpha-\beta) n} I_{s / r}^{(2)}(h n, j n, k n, l n, m n)=B_{n}
\end{array}\right.
$$

with $A_{n}, B_{n} \in \mathbb{Z}$. 
At the end of Section 3 we have defined the level of a permutation $\chi \in \Phi$ (or of a left coset $\chi \Lambda$ ). Here we choose any permutation of level 2, e.g., the permutation $\boldsymbol{\varphi}$ in (3.15). By the invariance of $M, N, \alpha, \beta, \delta$ under the action of $\Phi$ we have, similarly to (4.4),

$$
\left\{\begin{array}{l}
d_{M n} d_{N n} s^{\alpha n}(s-r)^{\beta n} r^{(\delta-\alpha-\beta) n} \\
\quad \times I_{s / r}((m+h-k) n,(j+k-m) n, m n, \ln , k n)=A_{n}^{\prime}-B_{n}^{\prime} \operatorname{Li}_{2}(r / s) \\
\quad d_{M n} d_{N n} s^{\alpha n}(s-r)^{\beta n} r^{(\delta-\alpha-\beta) n} \\
\quad \times I_{s / r}^{(2)}((m+h-k) n,(j+k-m) n, m n, \ln , k n)=B_{n}^{\prime}
\end{array}\right.
$$

with $A_{n}^{\prime}, B_{n}^{\prime} \in \mathbb{Z}$. Therefore, if we apply in (4.4) the transformation formula at the end of Section 3 corresponding to $\varphi$, we obtain

$$
\left\{\begin{array}{l}
A_{n}-B_{n} \operatorname{Li}_{2}(r / s)=\frac{(h n) !(j n) !}{((m+h-k) n) !((j+k-m) n) !}\left(A_{n}^{\prime}-B_{n}^{\prime} \operatorname{Li}_{2}(r / s)\right) \\
B_{n}=\frac{(h n) !(j n) !}{((m+h-k) n) !((j+k-m) n) !} B_{n}^{\prime},
\end{array}\right.
$$

whence, substituting the second equation in the first,

$$
\left\{\begin{array}{l}
((m+h-k) n) !((j+k-m) n) ! A_{n}=(h n) !(j n) ! A_{n}^{\prime} \\
((m+h-k) n) !((j+k-m) n) ! B_{n}=(h n) !(j n) ! B_{n}^{\prime} .
\end{array}\right.
$$

For any prime $p$, let

$$
\begin{aligned}
\zeta_{p} & =v_{p}(((m+h-k) n) !((j+k-m) n) !), \\
\eta_{p} & =v_{p}((h n) !(j n) !),
\end{aligned}
$$

where $v_{p}(W)$ denotes the exponential $p$-adic valuation of the integer $W>0$, i.e., the exponent of $p$ in the factorization of $W$ into powers of distinct primes. Since $v_{p}(W !)=[W / p]$ for $p>\sqrt{W}$, where $[x]$ denotes the integral part of $x$, from the definition (4.1) we infer that for any prime $p>\sqrt{M n}$ we have

$$
\begin{aligned}
\zeta_{p} & =\left[\frac{(m+h-k) n}{p}\right]+\left[\frac{(j+k-m) n}{p}\right], \\
\eta_{p} & =\left[\frac{h n}{p}\right]+\left[\frac{j n}{p}\right] .
\end{aligned}
$$

Let

$$
\omega=\{n / p\}=n / p-[n / p]
$$


be the fractional part of $n / p$. Following the arguments in [4] pp. 44-45, we get

$$
-1 \leq \zeta_{p}-\eta_{p}=[(m+h-k) \omega]+[(j+k-m) \omega]-[h \omega]-[j \omega] \leq 1 .
$$

Thus, removing the primes $p>\sqrt{M n}$ dividing the factorials on both sides of each equation (4.5), we conclude as in [4] p. 45 that any prime $p>\sqrt{M n}$ for which

$$
[(m+h-k) \omega]+[(j+k-m) \omega]<[h \omega]+[j \omega]
$$

divides $A_{n}$ and $B_{n}$.

A similar discussion applies to the transformation formulae, listed at the end of Section 3, corresponding to the permutations $\lambda \varphi$ and $\varphi \lambda \varphi \lambda \varphi$, which are also of level 2 . We now consider a permutation in $\Phi$ of level 3, e.g., $\varphi \lambda \varphi$. Again by Theorem 2.1 we obtain

$$
\left\{\begin{array}{l}
d_{M n} d_{N n} s^{\alpha n}(s-r)^{\beta n} r^{(\delta-\alpha-\beta) n} \\
\times I_{s / r}((h+j-l) n,(l+m-j) n,(j+k-m) n, m n, l n)=A_{n}^{\prime \prime}-B_{n}^{\prime \prime} \operatorname{Li}_{2}(r / s) \\
d_{M n} d_{N n} s^{\alpha n}(s-r)^{\beta n} r^{(\delta-\alpha-\beta) n} \\
\times I_{s / r}^{(2)}((h+j-l) n,(l+m-j) n,(j+k-m) n, m n, l n)=B_{n}^{\prime \prime}
\end{array}\right.
$$

with $A_{n}^{\prime \prime}, B_{n}^{\prime \prime} \in \mathbb{Z}$. Hence, applying in (4.4) the transformation formula corresponding to $\varphi \lambda \varphi$ and arguing as above, we get

$$
\left\{\begin{aligned}
((h+j-l) n) !((l+m-j) n) !((j+k & -m) n) ! A_{n} \\
& =(h n) !(j n) !(k n) ! A_{n}^{\prime \prime} \\
((h+j-l) n) !((l+m-j) n) !((j+k & -m) n) ! B_{n} \\
& =(h n) !(j n) !(k n) ! B_{n}^{\prime \prime} .
\end{aligned}\right.
$$

Let, for any prime $p$,

$$
\begin{aligned}
\vartheta_{p} & =v_{p}(((h+j-l) n) !((l+m-j) n) !((j+k-m) n) !), \\
\xi_{p} & =v_{p}((h n) !(j n) !(k n) !) .
\end{aligned}
$$

Then, as in [4] p. 46, for $p>\sqrt{M n}$ we have

$$
\begin{aligned}
\vartheta_{p}-\xi_{p} & =[(h+j-l) \omega]+[(l+m-j) \omega]+[(j+k-m) \omega]-[h \omega]-[j \omega]-[k \omega] \\
& =V_{1}+V_{2},
\end{aligned}
$$

with

$$
\begin{aligned}
& V_{1}=[(h+j-l) \omega]+[(l+m-j) \omega]-[h \omega]-[m \omega], \\
& V_{2}=[m \omega]+[(j+k-m) \omega]-[j \omega]-[k \omega]
\end{aligned}
$$


satisfying $-1 \leq V_{1} \leq 1$ and $-1 \leq V_{2} \leq 1$. Therefore $-2 \leq \vartheta_{p}-\xi_{p} \leq$ 2. Removing the primes $p>\sqrt{M n}$ dividing the factorials on both sides of each equation (4.7), we conclude as in [4] pp. 46-47 that any prime $p>\sqrt{M n}$ for which

$$
[(h+j-l) \omega]+[(l+m-j) \omega]+[(j+k-m) \omega]<[h \omega]+[j \omega]+[k \omega]
$$

divides $A_{n}$ and $B_{n}$, and any prime $p>\sqrt{M n}$ for which

$$
\left\{\begin{array}{l}
{[(h+j-l) \omega]+[(l+m-j) \omega]<[h \omega]+[m \omega]} \\
{[m \omega]+[(j+k-m) \omega]<[j \omega]+[k \omega]}
\end{array}\right.
$$

is such that $p^{2}$ divides $A_{n}$ and $B_{n}$.

From (4.6), (4.8), (4.9), and the similar inequalities arising from the other permutations in $\boldsymbol{\Phi}$ of levels 2 and 3, we obtain the following

Lemma 4.1. Let $\omega=\{n / p\}$. Any prime $p>\sqrt{M n}$ satisfying at least one of the following five inequalities:

$$
\begin{aligned}
& {[(m+h-k) \omega]+[(j+k-m) \omega]<[h \omega]+[j \omega],} \\
& {[(h+j-l) \omega]+[(l+m-j) \omega]<[h \omega]+[m \omega],} \\
& {[(l+m-j) \omega]+[(j+k-m) \omega]<[k \omega]+[l \omega],} \\
& {[(h+j-l) \omega]+[(l+m-j) \omega]+[(j+k-m) \omega]<[h \omega]+[j \omega]+[k \omega],} \\
& {[(m+h-k) \omega]+[(j+k-m) \omega]+[(l+m-j) \omega]<[h \omega]+[l \omega]+[m \omega]}
\end{aligned}
$$

divides the integers $A_{n}$ and $B_{n}$ in (4.4).

Any prime $p>\sqrt{M n}$ satisfying at least one of the following two conditions:

$$
\begin{aligned}
& \left\{\begin{array}{l}
{[(h+j-l) \omega]+[(l+m-j) \omega]<[h \omega]+[m \omega] \text { and }} \\
{[m \omega]+[(j+k-m) \omega]<[j \omega]+[k \omega],}
\end{array}\right. \\
& \left\{\begin{array}{l}
{[(m+h-k) \omega]+[(j+k-m) \omega]<[h \omega]+[j \omega] \text { and }} \\
{[j \omega]+[(l+m-j) \omega]<[l \omega]+[m \omega]}
\end{array}\right.
\end{aligned}
$$

is such that $p^{2}$ divides $A_{n}$ and $B_{n}$.

Let $\Omega$ be the set of real numbers $\omega \in[0,1)$ satisfying at least one of the five inequalities (4.10), and let $\Omega^{\prime}$ be the set of real numbers $\omega \in[0,1)$ satisfying at least one of the two conditions (4.11). Since each of (4.11) implies one of the last two inequalities (4.10), we have $\Omega^{\prime} \subset \Omega$. Moreover, let

$$
\Delta_{n}=\prod_{\substack{p>\sqrt{M n} \\\{n / p\} \in \Omega}} p, \quad \Delta_{n}^{\prime}=\prod_{\substack{p>\sqrt{M n} \\\{n / p\} \in \Omega^{\prime}}} p \quad(n=1,2, \ldots),
$$


where $p$ denotes a prime. From Lemma 4.1 we get $\Delta_{n} \Delta_{n}^{\prime} \mid A_{n}$ and $\Delta_{n} \Delta_{n}^{\prime} \mid B_{n}$. Thus, if we define

$$
a_{n}=\frac{A_{n}}{\Delta_{n} \Delta_{n}^{\prime}}, \quad b_{n}=\frac{B_{n}}{\Delta_{n} \Delta_{n}^{\prime}},
$$

we have $a_{n}, b_{n} \in \mathbb{Z}$. Denoting

$$
D_{n}=\frac{d_{M n} d_{N n}}{\Delta_{n} \Delta_{n}^{\prime}}
$$

if we divide (4.4) by $\Delta_{n} \Delta_{n}^{\prime}$ we obtain

$$
\left\{\begin{array}{l}
D_{n} s^{\alpha n}(s-r)^{\beta n} r^{(\delta-\alpha-\beta) n} I_{s / r}(h n, j n, k n, \ln , m n)=a_{n}-b_{n} \operatorname{Li}_{2}(r / s) \\
D_{n} s^{\alpha n}(s-r)^{\beta n} r^{(\delta-\alpha-\beta) n} I_{s / r}^{(2)}(h n, j n, k n, \ln , m n)=b_{n} .
\end{array}\right.
$$

Since, by the prime number theorem, $d_{M n} d_{N n}=\exp ((M+N) n+o(n))$, we have

$$
\lim _{n \rightarrow \infty} \frac{1}{n} \log D_{n}=M+N-\lim _{n \rightarrow \infty} \frac{1}{n} \log \Delta_{n}-\lim _{n \rightarrow \infty} \frac{1}{n} \log \Delta_{n}^{\prime},
$$

and it is well known that

$$
\lim _{n \rightarrow \infty} \frac{1}{n} \log \Delta_{n}=\int_{\Omega} \mathrm{d} \psi(x), \quad \lim _{n \rightarrow \infty} \frac{1}{n} \log \Delta_{n}^{\prime}=\int_{\Omega^{\prime}} \mathrm{d} \psi(x),
$$

where $\psi(x)=\Gamma^{\prime}(x) / \Gamma(x)$ is the logarithmic derivative of the Euler gammafunction (see [4] p. 51, or [7] pp. 463-464 for a full proof). Hence

$$
\lim _{n \rightarrow \infty} \frac{1}{n} \log D_{n}=M+N-\left(\int_{\Omega} \mathrm{d} \psi(x)+\int_{\Omega^{\prime}} \mathrm{d} \psi(x)\right) .
$$

We remark that in order to get a good asymptotic behaviour, as $n \rightarrow \infty$, of $I_{s / r}(h n, j n, k n, l n, m n)$ and $I_{s / r}^{(2)}(h n, j n, k n, l n, m n)$ in (4.12), a natural choice of the parameters would be $j=k=l=m$. However, with this choice the inequalities (4.10) become $[h \omega]+[j \omega]<[h \omega]+[j \omega], 2[j \omega]<2[j \omega]$ and $[h \omega]+2[j \omega]<[h \omega]+2[j \omega]$, and therefore are all false, whence $\Omega=\varnothing$. Thus the arithmetical correction

$$
\int_{\Omega} \mathrm{d} \psi(x)+\int_{\Omega^{\prime}} \mathrm{d} \psi(x)
$$

in (4.13) vanishes for $j=k=l=m$. As a consequence, the qualitative and quantitative irrationality results for $\operatorname{Li}_{2}(r / s)$ that can be obtained with the choice $j=k=l=m$ are worse than those given by suitable choices of the parameters yielding a positive arithmetical correction (4.14). 


\section{The irrationality measure of $\operatorname{Li}_{2}(r / s)$}

We henceforth assume the integers $h, j, k, l, m$ to be $>0$, and such that $l+m-j$, $m+h-k, h+j-l, j+k-m$ are also $>0$. We seek the stationary points, with respect to $x, y$, of the function

$$
f_{z}(x, y):=\frac{x^{j}(1-x)^{h} y^{k}(1-y)^{l}}{(x(1-y)+y z)^{j+k-m}} \quad(z>1)
$$

for which $x(1-x) y(1-y) \neq 0$. Since

$$
\begin{aligned}
& \frac{1}{f_{z}} \frac{\partial f_{z}}{\partial x}=\frac{\partial}{\partial x} \log f_{z}=\frac{j}{x}-\frac{h}{1-x}-\frac{(j+k-m)(1-y)}{x(1-y)+y z}, \\
& \frac{1}{f_{z}} \frac{\partial f_{z}}{\partial y}=\frac{\partial}{\partial y} \log f_{z}=\frac{k}{y}-\frac{l}{1-y}-\frac{(j+k-m)(z-x)}{x(1-y)+y z},
\end{aligned}
$$

we seek the solutions of the system

$$
\left\{\begin{array}{l}
\frac{1}{1-y}\left(\frac{j}{x}-\frac{h}{1-x}\right)=\frac{j+k-m}{x(1-y)+y z} \\
\frac{1}{z-x}\left(\frac{k}{y}-\frac{l}{1-y}\right)=\frac{j+k-m}{x(1-y)+y z} .
\end{array}\right.
$$

Equating the left sides of (5.2) and then solving with respect to $y$ yields

$$
y=\frac{k x(1-x)}{(k+l) x(1-x)+(z-x)(j-(h+j) x)} .
$$

If we substitute this into the first of (5.2), by a straightforward computation we obtain the cubic equation in $x$ :

$$
\begin{aligned}
U(x):=x((m+h-k) x+k-m)((h+j-l) x+l-j) & \\
& -z((h+j) x-j)((h+m) x-m)=0 .
\end{aligned}
$$

We have

$$
\begin{aligned}
U(0) & =-j m z<0, \\
U\left(\frac{j}{h+j}\right) & =\frac{h^{2} j l(j+k-m)}{(h+j)^{3}}>0, \\
U(1) & =-h^{2}(z-1)<0, \\
U(z) & =-z(z-1)((z-1)(l(m+h-k)+k(h+j))+h(k+l))<0,
\end{aligned}
$$


and the leading coefficient of $U(x)$ is $(m+h-k)(h+j-l)>0$. Hence $U(x)$ has three real roots $x_{0}, x_{1}, x_{2}$ satisfying

$$
0<x_{0}<\frac{j}{h+j}<x_{1}<1<z<x_{2} .
$$

If we set

$$
y_{v}=\frac{k x_{v}\left(1-x_{v}\right)}{(k+l) x_{v}\left(1-x_{v}\right)+\left(z-x_{v}\right)\left(j-(h+j) x_{v}\right)} \quad(v=0,1,2),
$$

by (5.3) we have the three stationary points $\left(x_{0}, y_{0}\right),\left(x_{1}, y_{1}\right),\left(x_{2}, y_{2}\right)$ of the function $f_{z}(x, y)$, satisfying $\partial f_{z} / \partial x=\partial f_{z} / \partial y=0, f_{z} \neq 0$.

From (5.6) we have $x_{0}>0,1-x_{0}>0, z-x_{0}>0$ and $j-(h+j) x_{0}>0$. Therefore (5.7) yields

$$
0<y_{0}<1
$$

In order to get further information on $y_{1}$ and $y_{2}$, it is convenient to go back to the system (5.2), and solve the second equation with respect to $x$. We get

$$
x=\frac{y z}{1-y} \frac{(l+m-j) y+j-m}{k-(l+m-j) y} .
$$

Substituting this into the first of (5.2), we easily obtain the cubic equation in $y$ :

$$
\begin{aligned}
V(y):=(z & -1) y((l+m-j) y-h-m)((l+m-j) y+j-m) \\
& +(l+m-j)(k+l-h) y^{2} \\
& +(h(m-j)-m(k+l)-k(l+m-j)) y+k m=0 .
\end{aligned}
$$

We have $V(0)=k m>0, V(1)=-l(h+j-l) z<0$, and the leading coefficient of $V(y)$ is $(z-1)(l+m-j)^{2}>0$. Hence $V(y)$ has three real roots, one of which is $<0$, one lies in $(0,1)$, and one is $>1$, and such roots must coincide with the values (5.7). Thus (5.9) yields

$$
x_{v}=\frac{y_{v} z}{1-y_{v}} \frac{(l+m-j) y_{v}+j-m}{k-(l+m-j) y_{v}} \quad(v=0,1,2),
$$

with the same $x_{v}, y_{v}$ as in (5.6) and (5.7) satisfying $U\left(x_{v}\right)=V\left(y_{v}\right)=0$. From (5.11) we obtain

$$
\frac{x_{v}}{x_{v}-z}=y_{v} \frac{(l+m-j) y_{v}+j-m}{(k+l) y_{v}-k}
$$

whence

$$
y_{v}-\frac{x_{v}}{x_{v}-z}=\frac{(j+k-m) y_{v}\left(y_{v}-1\right)}{(k+l) y_{v}-k}
$$


We know from (5.8) that $y_{0}$ is the root of $V(y)$ lying in $(0,1)$. If we temporarily denote by $y_{*}$ the root of $V(y)$ satisfying $y_{*}>1$ and by $x_{*}$ the corresponding root of $U(x)$ given by (5.11) for $y_{v}=y_{*}$, we have

$$
(l+m-j) y_{*}+j-m>l>0 \text { and }(k+l) y_{*}-k>l>0,
$$

whence, by (5.12),

$$
\frac{x_{*}}{x_{*}-z}>0
$$

By (5.6) we have $x_{*}>0$. Thus (5.14) yields $x_{*}>z$ whence, again by (5.6), $x_{*}=x_{2}, y_{*}=y_{2}$. From (5.13) we obtain

$$
y_{2}-\frac{x_{2}}{x_{2}-z}>0
$$

whence

$$
y_{2}>\frac{x_{2}}{x_{2}-z}>1
$$

Finally, the remaining root $y_{1}$ of $V(y)$ corresponding to $x_{1}$ must satisfy $y_{1}<0$. From (5.13) we get

$$
y_{1}-\frac{x_{1}}{x_{1}-z}<0
$$

whence, by (5.6),

$$
y_{1}<\frac{x_{1}}{x_{1}-z}<0
$$

We conclude that the three stationary points $\left(x_{0}, y_{0}\right),\left(x_{1}, y_{1}\right),\left(x_{2}, y_{2}\right)$ of the function (5.1), for which $x(1-x) y(1-y) \neq 0$, satisfy (5.6) and

$$
y_{1}<\frac{x_{1}}{x_{1}-z}<0<y_{0}<1<\frac{x_{2}}{x_{2}-z}<y_{2} \text {. }
$$

By our assumptions on $h, j, k, l, m$, the function $f_{z}(x, y)$ defined by (5.1) is clearly continuous on the compact unit square $\{0 \leq x \leq 1,0 \leq y \leq 1\}$, vanishes on the boundary and is positive inside. Hence the maximum of $f_{z}(x, y)$ in the unit square is attained at the stationary point $\left(x_{0}, y_{0}\right)$. As in Section 4 , we now replace $h, j, k, l$, $m$ by $h n, j n, k n, l n, m n$ respectively, where $h, j, k, l, m$ are fixed and $n=1,2, \ldots$. Then $f_{z}(x, y)$ is replaced by $f_{z}(x, y)^{n}$. The integral (2.1) becomes

$$
I_{z}^{(0)}(h n, j n, k n, l n, m n)=z^{-(l+m) n} \int_{0}^{1} \int_{0}^{1} f_{z}(x, y)^{n} \frac{\mathrm{d} x \mathrm{~d} y}{x(1-y)+y z},
$$


and by the simplest form of Laplace's asymptotic method we plainly have

$$
\lim _{n \rightarrow \infty} \frac{1}{n} \log I_{z}^{(0)}(h n, j n, k n, l n, m n)=-(l+m) \log z+\log f_{z}\left(x_{0}, y_{0}\right) .
$$

We now evaluate

$$
\limsup _{n \rightarrow \infty} \frac{1}{n} \log \left|I_{z}^{(v)}(h n, j n, k n, \ln , m n)\right| \quad(v=1,2) .
$$

Let $x$ be fixed, with $0 \leq x \leq 1$. On the circumference $|y-x /(x-z)|=\varrho$, the function

$$
\frac{|y|^{k}|1-y|^{l}}{|x(1-y)+y z|^{j+k-m+1}}
$$

equals

$$
\frac{|y|^{k}|1-y|^{l}}{(\varrho(z-x))^{j+k-m+1}}
$$

and hence is maximal at the point $y=x /(x-z)-\varrho$. Therefore

$$
\begin{gathered}
\left|\frac{1}{2 \pi i} \oint_{\left|y-\frac{x}{x-z}\right|=\varrho} \frac{y^{k}(1-y)^{l}}{(x(1-y)+y z)^{j+k-m+1}} \mathrm{~d} y\right| \\
\leq \frac{1}{z-x} \frac{\left(\varrho+\frac{x}{z-x}\right)^{k}\left(1+\varrho+\frac{x}{z-x}\right)^{l}}{(\varrho(z-x))^{j+k-m}} .
\end{gathered}
$$

Since the radius $\varrho$ is arbitrary, we can take the minimum of the right side of (5.17) when $\varrho>0$ varies. Such a minimum exists, because $k+l>j+k-m$ since $l+m-j>0$. Let $\varrho+x /(z-x)=v$, whence $v>x /(z-x)$ and $\varrho(z-x)=$ $v(z-x)-x=|x(1+v)-v z|$. Since $1 /(z-x) \leq 1 /(z-1)$, we see that the left side of (5.17) does not exceed

$$
\frac{1}{z-1} \min _{v>\frac{x}{z-x}} \frac{v^{k}(1+v)^{l}}{|x(1+v)-v z|^{j+k-m}} .
$$

Hence

$$
\begin{aligned}
& \left|\int_{0}^{1}\left(\frac{1}{2 \pi i} \oint_{\left|y-\frac{x}{x-z}\right|=\varrho} \frac{x^{j}(1-x)^{h} y^{k}(1-y)^{l}}{(x(1-y)+y z)^{j+k-m+1}} \mathrm{~d} y\right) \mathrm{d} x\right| \\
& \quad \leq \frac{1}{z-1} \max _{0 \leq x \leq 1} x^{j}(1-x)^{h} \min _{v>\frac{x}{z-x}} \frac{v^{k}(1+v)^{l}}{|x(1+v)-v z|^{j+k-m}} .
\end{aligned}
$$


With the change of variable $v=-y$ the right side of (5.18) becomes

$$
\frac{1}{z-1} \max _{0 \leq x \leq 1} \min _{y<\frac{x}{x-z}}\left|f_{z}(x, y)\right| .
$$

By (5.6) and (5.15),

$$
\max _{0 \leq x \leq 1} \min _{y<\frac{x}{x-z}}\left|f_{z}(x, y)\right|
$$

is attained at the saddle point $\left(x_{1}, y_{1}\right)$. It follows that

$$
\left|I_{z}^{(1)}(h, j, k, l, m)\right| \leq z^{-l-m} \frac{\left|f_{z}\left(x_{1}, y_{1}\right)\right|}{z-1} .
$$

Therefore, again replacing $h, j, k, l, m$ by $h n, j n, k n, l n, m n$,

$$
\begin{aligned}
\frac{1}{n} \log \mid I_{z}^{(1)}(h n, j n, k n, l n, & m n) \mid \\
& \leq-(l+m) \log z+\log \left|f_{z}\left(x_{1}, y_{1}\right)\right|-\frac{1}{n} \log (z-1) .
\end{aligned}
$$

We conclude that

$$
\begin{aligned}
\limsup _{n \rightarrow \infty} \frac{1}{n} \log \left|I_{z}^{(1)}(h n, j n, k n, \ln , m n)\right| & \\
& \leq-(l+m) \log z+\log \left|f_{z}\left(x_{1}, y_{1}\right)\right| .
\end{aligned}
$$

We now fix a point $x$ such that $|x-z|=\sigma$. For $|y-x /(x-z)|=\varrho$ we have

$$
|1-y| \leq\left|y-\frac{x}{x-z}\right|+\left|\frac{x}{x-z}-1\right|=\varrho+\frac{z}{\sigma}
$$

and

$$
|y| \leq 1+|y-1| \leq 1+\varrho+\frac{z}{\sigma}
$$

Consequently

$$
\frac{|y|^{k}|1-y|^{l}}{|x(1-y)+y z|^{j+k-m+1}} \leq \frac{\left(1+\varrho+\frac{z}{\sigma}\right)^{k}\left(\varrho+\frac{z}{\sigma}\right)^{l}}{(\varrho \sigma)^{j+k-m+1}} .
$$

It follows that

$$
\left|\frac{1}{2 \pi i} \oint_{\left|y-\frac{x}{x-z}\right|=\varrho} \frac{y^{k}(1-y)^{l}}{(x(1-y)+y z)^{j+k-m+1}} \mathrm{~d} y\right| \leq \frac{1}{\sigma} \frac{\left(1+\varrho+\frac{z}{\sigma}\right)^{k}\left(\varrho+\frac{z}{\sigma}\right)^{l}}{(\varrho \sigma)^{j+k-m}} .
$$


Since $z>1$, on the circumference $|x-z|=\sigma$ we plainly have $|x| \leq z+\sigma$ and $|1-x| \leq z+\sigma-1$. Therefore

$$
\begin{array}{r}
\left|\frac{1}{2 \pi i} \oint_{|x-z|=\sigma}\left(\frac{1}{2 \pi i} \oint_{\left|y-\frac{x}{x-z}\right|=\varrho} \frac{x^{j}(1-x)^{h} y^{k}(1-y)^{l}}{(x(1-y)+y z)^{j+k-m+1}} \mathrm{~d} y\right) \mathrm{d} x\right| \\
\leq \frac{(z+\sigma)^{j}(z+\sigma-1)^{h}(z+\sigma+\varrho \sigma)^{k}(z+\varrho \sigma)^{l}}{\sigma^{k+l}(\varrho \sigma)^{j+k-m}}
\end{array}
$$

for any $\varrho, \sigma>0$. Hence, denoting $\sigma=u$ and $\varrho \sigma=v$, the left side of (5.20) does not exceed

$$
\min _{u, v>0} \frac{(z+u)^{j}(z+u-1)^{h}(z+u+v)^{k}(z+v)^{l}}{u^{k+l} v^{j+k-m}} .
$$

The minimum exists since the above function of $u$ and $v$ is easily seen to be large outside a compact subset of $\{u>0, v>0\}$.

With the change of variables $u=x-z$ and $v=-x(1-y)-y z,(5.21)$ becomes

$$
\min _{\substack{x>z \\ y>\frac{x}{x-z}}}\left|\frac{x^{j}(1-x)^{h} y^{k}(x-z)^{k}(1-y)^{l}(x-z)^{l}}{(x-z)^{k+l}(x(1-y)+y z)^{j+k-m}}\right|=\min _{\substack{x>z \\ y>\frac{x}{x-z}}}\left|f_{z}(x, y)\right| .
$$

By (5.6) and (5.15) this minimum is attained at $\left(x_{2}, y_{2}\right)$. Therefore

$$
\left|I_{z}^{(2)}(h, j, k, l, m)\right| \leq z^{-l-m}\left|f_{z}\left(x_{2}, y_{2}\right)\right|,
$$

whence

$$
\begin{aligned}
\limsup _{n \rightarrow \infty} \frac{1}{n} \log \mid I_{z}^{(2)}(h n, j n, k n, \ln , & m n) \mid \\
& \leq-(l+m) \log z+\log \left|f_{z}\left(x_{2}, y_{2}\right)\right|
\end{aligned}
$$

As in Section 4, we now take $z=s / r$ with $r, s \in \mathbb{Z}, 1 \leq r<s$. In order to get from the above estimates an irrationality measure of $\operatorname{Li}_{2}(r / s)$, we assume that $h, j, k, l, m$ are chosen so that at the stationary points $\left(x_{0}, y_{0}\right)$ and $\left(x_{1}, y_{1}\right)$ of $f_{z}(x, y)=f_{s / r}(x, y)$ we have

$$
f_{s / r}\left(x_{0}, y_{0}\right)>\left|f_{s / r}\left(x_{1}, y_{1}\right)\right|
$$

We apply to (4.12) the following standard lemma (see [4], Lemma 4.3): 
Lemma 5.1. Let $L \in \mathbb{R}$, and let $\left(a_{n}\right),\left(b_{n}\right)$ be sequences of integers satisfying

$$
\lim _{n \rightarrow \infty} \frac{1}{n} \log \left|a_{n}-b_{n} L\right|=-A
$$

and

$$
\limsup _{n \rightarrow \infty} \frac{1}{n} \log \left|b_{n}\right| \leq B
$$

for some positive numbers $A$ and $B$. Then $L \notin \mathbb{Q}$, and

$$
\mu(L) \leq \frac{B}{A}+1
$$

From the first equation in (4.12) and the definition (2.4) we have

$$
\begin{array}{r}
\left|a_{n}-b_{n} \mathrm{Li}_{2}(r / s)\right|=D_{n} s^{\alpha n}(s-r)^{\beta n} r^{(\delta-\alpha-\beta) n} I_{s / r}^{(0)}(h n, j n, k n, l n, m n) \\
\times\left|1-\log (s / r) \frac{I_{s / r}^{(1)}(h n, j n, k n, l n, m n)}{I_{s / r}^{(0)}(h n, j n, k n, l n, m n)}\right| .
\end{array}
$$

By (5.16), (5.19) and the assumption (5.23) we get

$$
\lim _{n \rightarrow \infty} \frac{I_{s / r}^{(1)}(h n, j n, k n, l n, m n)}{I_{s / r}^{(0)}(h n, j n, k n, l n, m n)}=0 .
$$

Therefore, applying (4.13) and (5.16) in (5.24),

$$
\begin{aligned}
\lim _{n \rightarrow \infty} \frac{1}{n} \log \mid a_{n}-b_{n} & \operatorname{Li}_{2}(r / s) \mid=M+N-\left(\int_{\Omega} \mathrm{d} \psi(x)+\int_{\Omega^{\prime}} \mathrm{d} \psi(x)\right) \\
& +(\alpha-l-m) \log s+(\delta-\alpha-\beta+l+m) \log r \\
& +\beta \log (s-r)+\log f_{s / r}\left(x_{0}, y_{0}\right) .
\end{aligned}
$$

Similarly, from the second equation in (4.12), from (4.13) and (5.22) we obtain

$$
\begin{aligned}
\limsup _{n \rightarrow \infty} \frac{1}{n} \log \left|b_{n}\right| \leq & M+N-\left(\int_{\Omega} \mathrm{d} \psi(x)+\int_{\Omega^{\prime}} \mathrm{d} \psi(x)\right) \\
& +(\alpha-l-m) \log s+(\delta-\alpha-\beta+l+m) \log r \\
& +\beta \log (s-r)+\log \left|f_{s / r}\left(x_{2}, y_{2}\right)\right| .
\end{aligned}
$$

By Lemma 2.8 we have, in any case,

$$
\delta-\alpha-\beta+l+m=m+h-k .
$$


For brevity, let

$$
c_{0}=-\log f_{s / r}\left(x_{0}, y_{0}\right), \quad c_{1}=-\log \left|f_{s / r}\left(x_{1}, y_{1}\right)\right|, \quad c_{2}=\log \left|f_{s / r}\left(x_{2}, y_{2}\right)\right|,
$$

so that the assumption (5.23) is $c_{0}<c_{1}$, and let

$$
\begin{aligned}
c_{3}=M+N & -\left(\int_{\Omega} \mathrm{d} \psi(x)+\int_{\Omega^{\prime}} \mathrm{d} \psi(x)\right) \\
& +(\alpha-l-m) \log s+(m+h-k) \log r+\beta \log (s-r) .
\end{aligned}
$$

From Lemma 5.1 and from (5.25), (5.26) and (5.27) we obtain the following theorem.

Theorem 5.2. With the above notation and assumptions, if $c_{3}<c_{0}<c_{1}$ then $\mathrm{Li}_{2}(r / s) \notin \mathbb{Q}$ and

$$
\mu\left(\operatorname{Li}_{2}(r / s)\right) \leq \frac{c_{2}+c_{3}}{c_{0}-c_{3}}+1=\frac{c_{0}+c_{2}}{c_{0}-c_{3}} .
$$

We remark that, owing to (5.27), the irrationality measure of $\mathrm{Li}_{2}(r / s)$ in Theorem 5.2 is independent of $\delta$, i.e. of an upper bound for $\frac{1}{n} \max \left\{\operatorname{deg} P_{n}, \operatorname{deg} Q_{n}\right\}$, where $P_{n}$ and $Q_{n}$ are the polynomials in (4.3).

\section{Numerical results}

In this section we give some applications of Theorem 5.2. For this purpose, we first require upper and lower bounds for the roots of the polynomials $U(x)$ and $V(y)$ improving upon (5.6) and (5.15) when $z$ is large. We see in (5.4) that $U(x)$ is invariant under the action of the permutation $\lambda$ given by (3.2). Therefore, from the value of $U(j /(h+j))$ in (5.5) we get, by applying $\lambda$,

$$
U\left(\frac{m}{h+m}\right)=\frac{h^{2} k m(l+m-j)}{(h+m)^{3}}>0 .
$$

Hence, by (5.6), both $j /(h+j)$ and $m /(h+m)$ lie between the roots $x_{0}$ and $x_{1}$ of $U(x)$.

From now on, we assume $j \neq m$. Then without loss of generality we may restrict our discussion to the case

$$
j<m
$$

for otherwise we apply the permutation $\lambda$ which interchanges $j$ and $m$. By (6.1) we have

$$
\frac{j}{h+j}<\frac{m}{h+m}
$$


Since $U(j /(h+j))$ and $U(m /(h+m))$ are independent of $z$, an easy argument involving $U^{\prime}(x)$ and $U^{\prime \prime}(x)$ yields

$$
0<\frac{j}{h+j}-x_{0}=O(1 / z) \quad(z \rightarrow+\infty)
$$

and

$$
0<x_{1}-\frac{m}{h+m}=O(1 / z) \quad(z \rightarrow+\infty)
$$

Using the leading coefficient and the constant term of $U(x)$, for the third root $x_{2}$ of $U(x)$ we get, by (6.2) and (6.3),

$$
x_{2}=\frac{j m z}{(m+h-k)(h+j-l) x_{0} x_{1}}=\frac{(h+j)(h+m)}{(m+h-k)(h+j-l)} z+O(1) .
$$

On the other hand, a straightforward computation shows that, for any $z>1$,

$$
U\left(\frac{(h+j)(h+m)}{(m+h-k)(h+j-l)} z\right)>0
$$

Therefore, by (5.6),

$$
0<\frac{(h+j)(h+m)}{(m+h-k)(h+j-l)} z-x_{2}=O(1) \quad(z \rightarrow+\infty) .
$$

A similar elementary discussion gives asymptotic estimates for the roots of the polynomial $V(y)$ defined in (5.10). Since

$$
V\left(\frac{m-j}{l+m-j}\right)=\frac{j l(j+k-m)}{l+m-j}>0
$$

and

$$
V\left(\frac{h+m}{l+m-j}\right)=-\frac{h(m+h-k)(h+j-l)}{l+m-j}<0
$$

are independent of $z$, and since

$$
y_{1}=-\frac{k m}{(z-1)(l+m-j)^{2} y_{0} y_{2}},
$$

we easily obtain

$$
0<y_{0}-\frac{m-j}{l+m-j}=O(1 / z),
$$




$$
\begin{gathered}
0<y_{2}-\frac{h+m}{l+m-j}=O(1 / z), \\
0<y_{1}+\frac{k m}{(h+m)(m-j)(z-1)}=O\left(1 / z^{2}\right),
\end{gathered}
$$

for $z \rightarrow+\infty$.

In turn, such estimates for the roots of $U(x)$ and $V(y)$ yield asymptotic formulae for the quantities $c_{0}, c_{1}$ and $c_{2}$ defined by (5.28) as $z=s / r \rightarrow+\infty$ with $r \geq 1$ fixed. We have

$$
\begin{aligned}
c_{0}=-j \log x_{0}-h \log \left(1-x_{0}\right)-k \log y_{0}-l \log \left(1-y_{0}\right) & +(j+k-m) \log \left(x_{0}\left(1-y_{0}\right)+y_{0} z\right) \\
= & (j+k-m) \log z+O(1), \\
c_{1}=-j \log x_{1}-h \log \left(1-x_{1}\right)-k \log \left|y_{1}\right|-l \log \left(1-y_{1}\right) & \quad+(j+k-m) \log \left|x_{1}\left(1-y_{1}\right)+y_{1} z\right| \\
= & k \log z+O(1), \quad-(j+k-m) \log \left|x_{2}\left(1-y_{2}\right)+y_{2} z\right| \\
c_{2}= & j \log x_{2}+h \log \left|1-x_{2}\right|+k \log y_{2}+l \log \left|1-y_{2}\right| \\
= & (m+h-k) \log z+O(1) .
\end{aligned}
$$

Finally, by (5.29) with $s / r=z, r$ fixed and $s \rightarrow+\infty$,

$$
c_{3}=(\alpha+\beta-l-m) \log z+O(1) .
$$

From (6.8) and (6.9) we get

$$
c_{1}-c_{0}=(m-j) \log z+O(1),
$$

whence, by (6.1), $c_{1}-c_{0}>0$ for any sufficiently large $z$. Also, all the constants implied by the $O$-symbols in the preceding asymptotic formulae can be explicitly and easily computed. Therefore, by (6.8), (6.10), (6.11) and Theorem 5.2, we obtain the following

Corollary 6.1. Let $h, j, k, l, m>0$ be integers satisfying $l+m-j, m+h-k$, $h+j-l, j+k-m>0, j<m$, and

$$
\alpha+\beta<j+k+l,
$$

where $\alpha$ and $\beta$ are defined by (2.9). For any fixed integer $r \geq 1$ there exist effectively computable constants $s_{1}=s_{1}(r)>r$ and $C=C(r)>0$ (both depending also on $h, j, k, l, m)$ such that for any integer $s \geq s_{1}$ we have $\operatorname{Li}_{2}(r / s) \notin \mathbb{Q}$, with

$$
\mu\left(\operatorname{Li}_{2}(r / s)\right)<\frac{h+j}{j+k+l-\alpha-\beta}\left(1+\frac{C}{\log s}\right) .
$$


In the sequel we describe an effective algorithm, implicitly given in Corollary 6.1, which allows us to compute, for any integer $r \geq 1$, an admissible value for the above constant $s_{1}(r)$, and an irrationality measure of $\operatorname{Li}_{2}(r / s)$ depending on $r$ but not on $s$ and valid for all integers $s \geq s_{1}(r)$. Naturally, for suitably fixed $s$ (not necessarily $\geq s_{1}(r)$ ) the values of $h, j, k, l, m$ used in the algorithm for the given $r$ can be modified, in order to improve the irrationality measure of $\operatorname{Li}_{2}(r / s)$ for the special value of $s$ chosen if $s \geq s_{1}(r)$, or to give a new irrationality measure of $\operatorname{Li}_{2}(r / s)$ if $s<s_{1}(r)$.

For any fixed $r$, the algorithm consists of the following three steps.

1. We choose integers $h, j, k, l, m$ satisfying

$$
\begin{gathered}
h, j, k, l, m>0, \\
l+m-j, m+h-k, h+j-l, j+k-m>0, \\
j<m, \\
k+l-h \leq 0
\end{gathered}
$$

whence $\beta=0$, and

$$
\alpha=l+m,
$$

so that (6.12) holds. Also, by (5.29), (6.16) and (6.17),

$$
c_{3}=M+N-\left(\int_{\Omega} \mathrm{d} \psi(x)+\int_{\Omega^{\prime}} \mathrm{d} \psi(x)\right)+(m+h-k) \log r
$$

is a constant independent of $s$.

2. In view of the asymptotic formulae (6.2), (6.3), (6.4), (6.5), (6.6), (6.7), using the values $h, j, k, l, m$ in Step 1 we compute constants $\xi_{0}, \eta_{0}, \xi_{1}, \eta_{1}, \xi_{2}, \eta_{2}>0$ and $z_{1}>1$ such that for any $z \geq z_{1}$ we have

$$
\begin{gathered}
\frac{j}{h+j}-\frac{\xi_{0}}{z}<x_{0}<\frac{j}{h+j}, \\
\frac{m-j}{l+m-j}<y_{0}<\frac{m-j}{l+m-j}+\frac{\eta_{0}}{z-1}, \\
\frac{m}{h+m}<x_{1}<\frac{m}{h+m}+\frac{\xi_{1}}{z},
\end{gathered}
$$




$$
\begin{gathered}
-\frac{k m}{(h+m)(m-j)(z-1)}<y_{1}<-\frac{k m}{(h+m)(m-j)\left(z-1+\eta_{1}\right)}, \\
\frac{(h+j)(h+m)}{(m+h-k)(h+j-l)} z-\xi_{2}<x_{2}<\frac{(h+j)(h+m)}{(m+h-k)(h+j-l)} z, \\
\frac{h+m}{l+m-j}<y_{2}<\frac{h+m}{l+m-j}+\frac{\eta_{2}}{z-1} .
\end{gathered}
$$

Next, using these upper and lower bounds in (6.8), (6.9) and (6.10), we compute numerical approximations $c_{0}^{\prime}, c_{0}^{\prime \prime}$ of $c_{0} ; c_{1}^{\prime}$ of $c_{1} ; c_{2}^{\prime \prime}$ of $c_{2}$, all depending on $z$, such that for any $z \geq z_{1}$ we have

$$
c_{3}<c_{0}^{\prime}<c_{0}<c_{0}^{\prime \prime}<c_{1}^{\prime}<c_{1} \text { and } c_{2}<c_{2}^{\prime \prime},
$$

whence the assumptions of Theorem 5.2 hold, and such that

$$
\frac{c_{0}^{\prime}+c_{2}^{\prime \prime}}{c_{0}^{\prime}-c_{3}}
$$

is a decreasing function of $z$. Thus, by Theorem 5.2, for any integer $s \geq s_{1}(r):=$ $z_{1} r$ we obtain $\operatorname{Li}_{2}(r / s) \notin \mathbb{Q}$, with

$$
\mu\left(\operatorname{Li}_{2}(r / s)\right)<\left[\frac{c_{0}^{\prime}+c_{2}^{\prime \prime}}{c_{0}^{\prime}-c_{3}}\right]_{z=z_{1}} .
$$

3. We find integers $s_{0}(r)$ and $s_{2}(r)$ with $r<s_{0}(r) \leq s_{1}(r) \leq s_{2}(r)$, and for each $s=s_{0}(r), s_{0}(r)+1, \ldots, s_{2}(r)$ we compute ex novo $h, j, k, l, m$ depending on $s$ and satisfying (6.13) and (6.14), but not necessarily (6.15), (6.16) or (6.17), so that, by a direct application of Theorem 5.2, for any integer $s$ with $s_{0}(r) \leq$ $s \leq s_{2}(r)$ we obtain $\operatorname{Li}_{2}(r / s) \notin \mathbb{Q}$ together with a good irrationality measure of $\operatorname{Li}_{2}(r / s)$, improving upon (6.19) for all $s$ with $s_{1}(r) \leq s \leq s_{2}(r)$.

Here we give some examples of the above algorithm, by explicitly treating the cases $r=1,2,3,4$.

Case $r=1$.

(Step 1) We take

$$
h=40, \quad j=20, k=25, \quad l=15, \quad m=31,
$$

whence

$$
c_{3}=75.10604 \ldots
$$


(Step 2) We find

$$
\xi_{0}=0.08, \quad \eta_{0}=0.25, \quad \xi_{1}=0.21, \quad \eta_{1}=1, \quad \xi_{2}=0.55, \quad \eta_{2}=0.75,
$$

with $z_{1}=15$, and we compute $c_{0}^{\prime}, c_{0}^{\prime \prime} ; c_{1}^{\prime}$; $c_{2}^{\prime \prime}$, satisfying (6.18) for $z \geq 15$, such that

$$
\left[\frac{c_{0}^{\prime}+c_{2}^{\prime \prime}}{c_{0}^{\prime}-c_{3}}\right]_{z=15}=16.13928 \ldots
$$

Therefore, by (6.19), we get $\operatorname{Li}_{2}(1 / s) \notin \mathbb{Q}$ and

$$
\mu\left(\operatorname{Li}_{2}(1 / s)\right)<16.13928 \ldots
$$

for all integers $s \geq 15$.

(Step 3) We find $s_{0}(1)=6$, and choose $s_{2}(1)=20$. For $s=6$ we take the following values:

$$
h=98, \quad j=49, \quad k=71, \quad l=34, \quad m=91,
$$

whence

$$
\begin{gathered}
l+m-j=76, \quad m+h-k=118, \quad h+j-l=113, \quad j+k-m=29, \\
M=m+h-k=118, \quad N=h+j-l=113, \\
\alpha=125, \quad \beta=7, \\
\int_{\Omega} \mathrm{d} \psi(x)=42.71230 \ldots, \quad \int_{\Omega^{\prime}} \mathrm{d} \psi(x)=1.30962 \ldots, \\
c_{3}=198.24413 \ldots, \\
c_{0}=199.01324 \ldots, \quad c_{1}=199.20868 \ldots, \quad c_{2}=403.42718 \ldots,
\end{gathered}
$$

so that, by Theorem 5.2, $\operatorname{Li}_{2}(1 / 6) \notin \mathbb{Q}$ and

$$
\mu\left(\operatorname{Li}_{2}(1 / 6)\right)<783.29036 \ldots
$$

For $6 \leq s \leq 20$ we find the following values: 


\begin{tabular}{|c||c|c|c|c|c||c|}
\hline$s$ & $h$ & $j$ & $k$ & $l$ & $m$ & $\mu\left(\mathrm{Li}_{2}(1 / s)\right)<$ \\
\hline \hline 6 & 98 & 49 & 71 & 34 & 91 & $783.29036 \ldots$ \\
\hline 7 & 80 & 40 & 58 & 29 & 72 & $69.68879 \ldots$ \\
\hline 8 & 106 & 53 & 72 & 36 & 90 & $47.42514 \ldots$ \\
\hline 9 & 92 & 46 & 59 & 32 & 75 & $30.43779 \ldots$ \\
\hline 10 & 74 & 37 & 49 & 24 & 59 & $24.04068 \ldots$ \\
\hline 11 & 70 & 35 & 46 & 24 & 55 & $19.79502 \ldots$ \\
\hline 12 & 40 & 20 & 25 & 15 & 31 & $17.59873 \ldots$ \\
\hline 13 & 110 & 55 & 69 & 41 & 84 & $16.29461 \ldots$ \\
\hline 14 & 152 & 76 & 94 & 57 & 114 & $15.12865 \ldots$ \\
\hline 15 & 62 & 31 & 39 & 23 & 46 & $14.39699 \ldots$ \\
\hline 16 & 98 & 49 & 60 & 38 & 72 & $13.70717 \ldots$ \\
\hline 17 & 66 & 33 & 40 & 26 & 48 & $13.17790 \ldots$ \\
\hline 18 & 68 & 34 & 42 & 26 & 49 & $12.71390 \ldots$ \\
\hline 19 & 70 & 35 & 43 & 27 & 50 & $12.30058 \ldots$ \\
\hline 20 & 72 & 36 & 44 & 28 & 51 & $11.94109 \ldots$ \\
\hline
\end{tabular}

These values should be compared with Hata's irrationality measures of $\mathrm{Li}_{2}(1 / s)$ for $7 \leq s \leq 18$ ([3], p. 386, Table 1).

Case $r=2$.

(Step 1) $\quad h=126, j=63, k=74, \quad l=52, \quad m=86$,

$$
c_{3}=234.72812 \ldots
$$

(Step 2) $\xi_{0}=0.14, \quad \eta_{0}=0.52, \quad \xi_{1}=0.28, \quad \eta_{1}=2, \quad \xi_{2}=0.58, \quad \eta_{2}=0.79$,

$$
\begin{gathered}
z_{1}=34, \\
{\left[\frac{c_{0}^{\prime}+c_{2}^{\prime \prime}}{c_{0}^{\prime}-c_{3}}\right]_{z=34}=74.21062 \ldots}
\end{gathered}
$$

Therefore, by (6.19), for any integer $s \geq 69$ we have $\operatorname{Li}_{2}(2 / s) \notin \mathbb{Q}$ and

$$
\mu\left(\operatorname{Li}_{2}(2 / s)\right)<74.21062 \ldots .
$$

(Step 3) We find $s_{0}(2)=51$, and choose $s_{2}(2)=71$. Thus we get

$$
\operatorname{Li}_{2}(2 / s) \notin \mathbb{Q} \text { for all integers } s \geq 51,
$$

and for $51 \leq s \leq 71$ we find the following values: 


\begin{tabular}{|c||c|c|c|c|c||c|}
\hline$s$ & $h$ & $j$ & $k$ & $l$ & $m$ & $\mu\left(\mathrm{Li}_{2}(2 / s)\right)<$ \\
\hline \hline 51 & 126 & 63 & 74 & 52 & 86 & $374.80498 \ldots$ \\
\hline 53 & 78 & 39 & 46 & 32 & 53 & $202.04426 \ldots$ \\
\hline 55 & 34 & 17 & 20 & 14 & 23 & $138.04296 \ldots$ \\
\hline 57 & 98 & 49 & 58 & 40 & 66 & $105.94462 \ldots$ \\
\hline 59 & 118 & 59 & 69 & 49 & 79 & $84.43554 \ldots$ \\
\hline 61 & 12 & 6 & 7 & 5 & 8 & $72.03610 \ldots$ \\
\hline 63 & 98 & 49 & 57 & 41 & 65 & $63.18738 \ldots$ \\
\hline 65 & 62 & 31 & 36 & 26 & 41 & $57.13550 \ldots$ \\
\hline 67 & 50 & 25 & 29 & 21 & 33 & $52.51391 \ldots$ \\
\hline 69 & 38 & 19 & 22 & 16 & 25 & $48.52733 \ldots$ \\
\hline 71 & 26 & 13 & 15 & 11 & 17 & $44.65752 \ldots$ \\
\hline
\end{tabular}

Case $r=3$.

For brevity, in this case and in the next we only give values for $s_{0}(r)$, and values of $h, j, k, l, m$ yielding an irrationality measure of $\mathrm{Li}_{2}(r / s)$ for $s=s_{0}(r)$.

For $r=3$ we find $s_{0}(3)=173$, so that

$\operatorname{Li}_{2}(3 / s) \notin \mathbb{Q}$ for all integers $s \geq 173$,

and, taking

$$
h=50, \quad j=25, \quad k=28, \quad l=22, \quad m=31,
$$

we get

$$
\mu\left(\operatorname{Li}_{2}(3 / 173)\right)<8872.70681 \ldots
$$

Case $r=4$.

Here we find $s_{0}(4)=423$, i.e.,

$$
\operatorname{Li}_{2}(4 / s) \notin \mathbb{Q} \text { for all integers } s \geq 423 \text {. }
$$

For

$$
h=68, \quad j=34, \quad k=37, \quad l=31, \quad m=40
$$

we get

$$
\mu\left(\operatorname{Li}_{2}(4 / 423)\right)<40596.78637 \ldots
$$




\section{References}

[1] F. BEUKERS, A note on the irrationality of $\zeta$ (2) and $\zeta$ (3), Bull. London Math. Soc. 11 (1979), 268-272.

[2] E. Bombieri, On G-functions, In: "Recent Progress in Analytic Number Theory", H. Halberstam and C. Hooley (eds.), Durham, 1979, Academic Press, London-New York, 1981, vol. 2, 1-67.

[3] M. Hata, Rational approximations to the dilogarithm, Trans. Amer. Math. Soc. 336 (1993), 363-387.

[4] G. Rhin and C. Viola, On a permutation group related to $\zeta(2)$, Acta Arith. 77 (1996), 23-56.

[5] G. Rhin and C. Viola, The group structure for $\zeta(3)$, Acta Arith. 97 (2001), 269-293.

[6] C. L. SiEgel, Über einige Anwendungen diophantischer Approximationen, Abh. Preuss. Akad. Wiss., 1929, n. 1; Gesammelte Abhandlungen, Band I, Springer-Verlag, BerlinHeidelberg, 1966, 209-266.

[7] C. Viola, On Siegel's method in diophantine approximation to transcendental numbers, Rend. Sem. Mat. Univ. Pol. Torino 53 (1995), 455-469.

Département de Mathématiques UFR MIM

Université de Metz

Ile du Saulcy

57045 Metz Cedex 01, France

rhin@ poncelet.univ-metz.fr

Dipartimento di Matematica

Università di Pisa

Largo B. Pontecorvo 5

56127 Pisa, Italy

viola@dm.unipi.it 